\title{
A SQP-SEMI-SMOOTH NEWTON-TYPE ALGORITHM APPLIED TO CONTROL OF THE INSTATIONARY NAVIER-STOKES SYSTEM SUBJECT TO CONTROL CONSTRAINTS
}

\author{
M. HINTERMÜLLER* AND M. HINZE ${ }^{\dagger}$
}

\begin{abstract}
Sequential quadratic programming (SQP) methods for the optimal control of the instationary Navier-Stokes equations with pointwise constraints on the control are considered. Due to the presence of the constraints the quadratic subproblems (QP) of SQP require a more sophisticated solver compared to the unconstrained case. In this paper, a semismooth Newton method is proposed for efficiently solving the QPs. The convergence analysis, which is performed in an appropriate function space setting, relies on the concept of slant differentiability for proving locally superlinear convergence of the QP-solver. For the analysis of the outer SQP-iteration a generalized equations approach is utilized. Sufficient conditions for guaranteeing strong regularity of the generalized equation are established which, in turn, allows to argue a quadratic rate of convergence of the SQP-method. The paper ends with a report on numerical results supporting the theoretical findings.
\end{abstract}

Key words. Box constraints, generalized equations, Navier-Stokes, optimal control, semismooth Newton, sequential quadratic programming.

AMS subject classifications. 90C55, 65K10, 49M37.

1. Introduction. In this paper we continue our efforts in devising efficient numerical algorithms for the optimal control of the instationary Navier-Stokes equations. While the contributions in $[21,24]$ focus on unconstrained problems with respect to the control, the goal of the present work is to extend the framework in [21] to problems involving box constraints on the control variable.

Throughout we focus on the problem

$$
\begin{array}{ll}
\text { minimize } J(y, u) & \text { over }(y, u) \in W \times U_{\text {ad }} \\
\text { subject to } & \text { in } Q=(0, T) \times \Omega, \\
\frac{\partial y}{\partial t}+(y \cdot \nabla) y-\nu \Delta y+\nabla p=u & \text { in } Q, \\
\operatorname{div} y=0 & \text { on } \Sigma=(0, T) \times \partial \Omega, \\
y(t, \cdot)=0 & \text { in } \Omega,
\end{array}
$$

with $U_{\text {ad }}$ denoting the closed convex subset of the Hilbert space $U$ of controls given by

$$
U_{\mathrm{ad}}=\{v \in U: a \leq v \leq b \text { a.e. in } Q\},
$$

where the bounds $a<b$ are sufficiently regular. Above $T>0$ denotes the finite time horizon. The space $W$ corresponding to the state variable (velocity field) $y$ and the choice of cost functionals $J$ will be specified below. The variable $u$ will be referred to as the control variable.

In the unconstrained case, i.e., $U_{\mathrm{ad}}=U$, the development of numerical techniques for the optimal control of the stationary as well as instationary Navier-Stokes

\footnotetext{
${ }^{*}$ Dept. of Computational and Applied Mathematics, Rice University, Houston, Texas, and University of Graz, Department of Mathematics, Heinrichstraße. 36, 8010 Graz, Austria (michael.hintermueller@uni-graz.at)

$\dagger$ Technical University of Dresden, Department of Mathematics, Dresden, Germany (hinze@math.tu-dresden.de)
} 
equations has received a considerable amount of attention. Here we refer to the monographs and selected papers $[3,6,11,14,16,17,18,21,24,28]$ covering distributed and boundary control problems. However, the presence of constraints on $u$ requires a different algorithmic approach and usually complicates the numerical treatment significantly. If one wishes to apply a gradient related approach (like, e.g., in [16]), then the fact that $U_{\text {ad }} \subset U$ requires Hilbert space projections and modifications in potential line search techniques for globalization. Recently, sequential quadratic programming (SQP) techniques have become feasible for solving the unconstrained version of (1.1); see for instance [18, 21, 24]. With respect to convergence speed the latter approach is typically superior to gradient methods. With regard to the computational complexity of SQP, the efficient solution of the linear-quadratic subproblems (QPs) is essential. In the presence of control constraints the QPs inherit the constraints from the problem formulation. As a consequence, compared to the unconstrained case more sophisticated QP-solvers have to be applied in order to compute appropriate search directions.

Motivated by the fast local convergence properties of SQP-techniques, we will extend the unconstrained approach of, e.g., $[21,24]$ to the control constrained case. The numerical QP-framework in this paper is related to the primal-dual active set strategy (pdAS) as introduced in [4] and further analyzed and tested in [5, 19, 20]. In particular, the latter results prove this method to be extremely efficient in case of control constraints. Recently, in [22] it was shown that the pdAS is a particular instance of a generalized Newton method for a class of optimization problems in function spaces. In the present context, we utilize an inexact version of pdAS which is due to the large size of the problem and in order to save computation time. This is in the spirit of inexact Newton techniques for smooth problems; see, e.g., [10, 15].

As a key result it will turn out that pdAS provides a framework which efficiently deals with the constraints of the type (1.2) and requires only a moderate number of modifications in a SQP-environment for unconstrained problems in order to include the constrained case, too. This is of particular advantage since it allows to extend one's favorite SQP-solver quite easily. The alterations needed for including constraints essentially comprise the storage of index sets referring to whether $u$ is equal to one of the bounds or not and changes in the conjugate gradient (CG) method for solving the linear systems arising in pdAS when solving the QP-problems. Moreover, if the initial control $u^{0}$ is feasible, i.e., $u^{0} \in U_{\text {ad }}$, and exact QP-solutions are computed, then the algorithm produces only feasible iterates with respect to the control variable.

Besides the numerical justification of our approach by a report on an excerpt of extensive numerical tests, an infinite dimensional convergence analysis for the SQPalgorithm is provided. By utilizing the concept of slant differentiability of a not necessarily (Fréchet) differentiable mapping between Banach spaces $[7,22]$ (see also [32] for a related notion), the locally superlinear convergence of the primal-dual active set algorithm (inner iteration) is established. For the analysis of the SQP-method (outer iteration) a generalized equations approach is utilized. In a different context, the potential of generalized equations for the analysis of algorithms for constrained optimal control problems was exploited previously in, e.g., $[1,12,30]$ and the references therein. Under a strong regularity property (see [27]) we prove that the SQP-iteration converges at a locally quadratic rate.

To the best of our knowledge, the only contribution dealing with control constrained optimal control of the instationary Navier-Stokes equations in a generalized Newton framework is given by [31]. In this paper, a Newton algorithm for computing 
a solution to the first order optimality conditions of the reduced problem

$$
\text { minimize } \quad \hat{J}(u)=J(y(u), u) \quad \text { subject to } u \in U_{\mathrm{ad}}
$$

is considered. Above $y(u)$ denotes the solution of the Navier-Stokes equations for given $u$. In [31] a locally superlinear rate of convergence of the generalized Newton method is established. Note the difference to our approach. Firstly, we use a SQPframework which requires a different convergence analysis yielding convergence at a locally quadratic rate. We utilize the pdAS, or equivalently a generalized Newton method, as the QP-solver and prove its locally superlinear convergence. In our tests, typically the pdAS terminates after 1-2 iterations with a solution fulfilling a stopping rule similar to inexact Newton methods. The stopping tolerance is tuned in such a way that fast progress of the outer iteration is maintained. Further, our generalized Newton method does not require a smoothing step for its analysis. And we use a different discretization concept which provides discrete, numerically computable controls without explicitly discretizing the controls. The controls are discretized implicitly through the optimality conditions in terms of the adjoint variables. For more details on this technique see [23].

The rest of the paper is organized as follows. In the next section we introduce the precise functional analytic setting of (1.1) and notation. Further we establish some basic results required for the convergence analysis. Section 3 is devoted to the first order optimality system for the underlying control problem. Due to the constraints the first order system involves a so-called complementarity system consisting of primal and dual inequality conditions and a nonlinear equality. Relying on the concept of complementarity functions, we reformulate the latter system as a set of nonsmooth equalities. In section 4 we introduce the reduced first order system and the SQPalgorithm. Due to the equality related to the complementarity function which is non-differentiable an active set type QP-solver is the focus of section 5. The section also includes the convergence analysis for the QP-solver. In the following section 6 local quadratic convergence of the SQP-iteration is established. In the final section 7 we provide a report on numerical results obtained by our SQP-method.

2. Preliminaries. For the convenience of the reader we collect the analytical preliminaries for a proper formulation of problem (1.1) and to establish convergence results for the algorithms presented in this paper. To define the spaces and operators required for the investigation of (1.1) we introduce the solenoidal spaces

$$
H=\left\{v \in C_{0}^{\infty}(\Omega)^{2}: \operatorname{div} v=0\right\}^{-\mid \cdot L_{L^{2}}}, V=\left\{v \in C_{0}^{\infty}(\Omega)^{2}: \operatorname{div} v=0\right\}^{-|\cdot|_{H^{1}}},
$$

with the superscripts denoting closures in the respective norms. We have (see [28])

$$
V \hookrightarrow H=H^{*} \hookrightarrow V^{*},
$$

with $\hookrightarrow$ denoting the continuous injection. The spaces $H^{*}$ and $V^{*}$ denote the dual spaces of $H$ and $V$, respectively. Further we define the Banach spaces

$$
W_{q}^{p}=\left\{v \in L^{p}(V): v_{t} \in L^{q}\left(V^{*}\right)\right\} \quad \text { and } \quad Z:=L^{2}(V) \times H,
$$

where $W_{q}^{p}$ is endowed with the norm

$$
|v|_{W_{q}^{p}}=|v|_{L^{p}(V)}+\left|v_{t}\right|_{L^{q}\left(V^{*}\right)},
$$


abbreviate

$$
W:=W_{2}^{2}
$$

and set $\langle\cdot, \cdot\rangle:=\langle\cdot, \cdot\rangle_{L^{2}\left(V^{*}\right), L^{2}(V)}$. Here $L^{2}(V)$ is an abbreviation for $L^{2}(0, T ; V)$ and similarly $L^{2}\left(V^{*}\right)=L^{2}\left(0, T ; V^{*}\right)$. Note that we also have $L^{2}(V) \hookrightarrow L^{2}(Q)^{2} \hookrightarrow L^{2}\left(V^{*}\right)$. We further need the following embedding result.

Proposition 2.1. Let $\epsilon \in(0,1)$. For all $1 \leq p<\frac{3}{2}+\frac{1+\epsilon}{1-\epsilon}=: \delta_{\epsilon}$ there holds

$$
W_{1+\epsilon}^{2} \hookrightarrow L^{p}(Q)^{2} .
$$

Proof. In [31, Lemma A1] the result is proved for $\epsilon=\frac{1}{3}$. It follows from $[2$, Theorem 1.1] that $W_{1+\epsilon}^{2} \hookrightarrow L^{q}(H)$ for all $q \in\left[1, \frac{4(1+\epsilon)}{1-\epsilon}\right)$. The remainder of the proof follows the lines of the proof of Lemma A1 in [31]. $\mathrm{c}$

We note that, up to a set of measure zero in $(0, T)$, elements $v \in W$ can be identified with elements in $C([0, T] ; H)$. In our convergence analysis we also need

$$
H^{2,1}(Q)=\left\{v \in L^{2}\left(0, T ; H^{2}(\Omega)\right): v_{t} \in L^{2}(H)\right\} .
$$

endowed with the norm

$$
|v|_{H^{2,1}}(Q):=|v|_{L^{2}\left(V \cap H^{2}(\Omega)^{2}\right)}^{2}+\left|v_{t}\right|_{L^{2}(H)}^{2} .
$$

In [29] (compare [26]) it is shown that for $\Omega \subset \mathbb{R}^{2}$

$$
H^{2,1}(Q) \hookrightarrow L^{\infty}\left(0, T ; H^{1}(\Omega)\right) \cap L^{q}(Q) \quad \text { for } \quad 1 \leq q<+\infty .
$$

In (1.2) $U$ denotes the Hilbert space of controls which is identified with its dual $U^{*}$. Throughout we adopt the frequent choice $U=L^{2}(Q)^{2}$. Note that $w \in L^{2}(0, T ; H)$ satisfies $w \in L^{2}(Q)^{2}$.

Concerning the class of cost functionals $J: W \times U \rightarrow \mathbb{R}$ considered herein, we invoke the following assumption.

Assumption 2.2.

- $J(y, u)=J_{1}(y)+J_{2}(u)$ is bounded from below, weakly lower semi-continuous and twice Fréchet differentiable with locally Lipschitzian second derivative.

- $J_{2}(u)=\frac{\alpha}{2}|u|_{U}^{2}$ which implies that $J$ is radially unbounded in $u$, i.e., $J(y, u) \rightarrow$ $\infty$ as $|u|_{U} \rightarrow \infty$, for every $y \in W$.

- $J_{1_{y}}(y), J_{1_{y y}}(y) v \in L^{1+\epsilon}\left(V^{*}\right) \cap W^{*}$ for all $v \in W$ and for some $\epsilon \in(0,1)$.

Our assumptions on $J$ are satisfied for the tracking type functional

$$
J(y, u)=\frac{1}{2} \int_{Q}|y-z|^{2} d x d t+\frac{\gamma}{2} \int_{\Omega}|y(T)-z(T)|^{2} d x+\frac{\alpha}{2}|u|_{U}^{2},
$$

and functionals involving the vorticity of the fluid like

$$
J(y, u)=\frac{1}{2} \int_{Q}\left|\nabla_{x} \times y(t, \cdot)\right|^{2} d x d t+\frac{\alpha}{2}|u|_{U}^{2},
$$

where $\alpha, \gamma>0$ and $z \in W$ are given. These two functionals are even infinitely Fréchet differentiable on $W \times U$. 
Associated with the governing equation in (1.1) we define the nonlinear mapping $e: W \times U \rightarrow Z^{*}$ by

$$
e(y, u)=\left(\frac{\partial y}{\partial t}+(y \cdot \nabla) y-\nu \Delta y-u, y(0)-y_{0}\right),
$$

where $y_{0} \in H$. Note that we could also include a class of operators $B$ acting on the control $u$ on the right hand side of the equation and in the first component in e. As long as $B$ fulfills certain regularity requirements, like in, e.g., [31], it poses no difficulty. In variational form the constraints in (1.1) can be equivalently expressed as: given $u \in U_{\text {ad }}$ find $y \in W$ such that $y(0)=y_{0}$ in $H$ and

$$
\left\langle y_{t}, v\right\rangle+\langle(y \cdot \nabla) y, v\rangle+\nu(\nabla y, \nabla v)_{L^{2}\left(L^{2}\right)}=\langle u, v\rangle \forall v \in L^{2}(V) .
$$

Utilizing $e$, the control problem (1.1) can be rewritten as

$$
\min _{(y, u) \in W \times U_{\mathrm{ad}}} J(y, u) \text { subject to } e(y, u)=0 \text { in } Z^{*} .
$$

For the analysis of the SQP-method we shall frequently refer to the variational solution of the linearized Navier-Stokes system and the adjoint equations in the solenoidal setting. For this purpose we state the following proposition which is proved in [21], compare also [24] for a similar analytic framework. It is also essential for Newton and quasi-Newton methods.

Proposition 2.3. Let $y \in W, v_{0} \in H$ and $g \in L^{2}\left(V^{*}\right)$. Then the system of linearized Navier-Stokes equations

$$
A(y) v=\left(g, v_{0}\right) \text { in } Z^{*} \Leftrightarrow\left\{\begin{array}{l}
v_{t}+(v \cdot \nabla) y+(y \cdot \nabla) v-\nu \Delta v=g \quad \text { in } L^{2}\left(V^{*}\right) \\
v(0)=v_{0} \text { in } H,
\end{array}\right.
$$

admits a unique variational solution $v \in W$. For $y \in W \cap L^{\infty}(V) \cap L^{2}\left(H^{2}(\Omega)^{2}\right)$, $v_{0} \in V$ and $g \in L^{2}(H)$ the unique solution $v$ of $(2.8)$ is an element of $H^{2,1}(Q)$ and satisfies the a-priori estimate

$$
|v|_{H^{2,1}(Q)} \leq C\left(|y|_{L^{\infty}(V)},|y|_{L^{2}\left(H^{2}(\Omega)^{2}\right)}\right)\left\{|g|_{L^{2}(H)}+\left|v_{0}\right|_{V}\right\} .
$$

Concerning the adjoint equation we state the following result.

Proposition 2.4. Let $y \in W$ and $f \in W^{*}$ then the adjoint equation

$$
A(y)^{*} w=f \quad \text { in } W^{*}
$$

admits a unique variational solution $w=\left(w^{1}, w^{0}\right) \in Z$. If $f \in L^{q}\left(V^{*}\right) \cap W^{*}(1 \leq q \leq$ $\infty)$, then for every $0 \leq \epsilon \leq \min \left\{q-1, \frac{1}{3}\right\}$ the function $w^{1}$ is an element of $W_{1+\epsilon}^{2}$ and the variational solution of

$$
\left\{\begin{array}{l}
-w_{t}^{1}+(\nabla y)^{\top} w^{1}-(y \cdot \nabla) w^{1}-\nu \Delta w^{1}=f \\
w^{1}(T)=0
\end{array}\right.
$$

and it satisfies $w^{1}(0)=w^{0}$. If in addition $y \in L^{\infty}(V)$ and $f \in L^{2}\left(V^{*}\right)$, then $w^{1} \in W$. For $y \in W \cap L^{\infty}(V) \cap L^{2}\left(H^{2}(\Omega)^{2}\right), v_{0} \in V$ and $f \in L^{2}(H)$ the unique solution $w^{1}$ of (2.9) is an element of $H^{2,1}(Q)$ and satisfies the a-priori estimate

$$
\left|w^{1}\right|_{H^{2,1}(Q)} \leq C\left(|y|_{L^{\infty}(V)},|y|_{L^{2}\left(H^{2}(\Omega)^{2}\right)}\right)|f|_{L^{2}(H)} .
$$

Further properties of the linearized and adjoint equations can be found in [24]. 
For the application of the SQP-method to (1.1) we need second order information of the Lagrangian $L$, which is defined below in (3.1). The basic ingredients are the derivatives of the operator $e$ which were characterized in [21], compare also [24]. For the convenience of the reader we state the following proposition.

Proposition 2.5. The operator $e=\left(e^{1}, e^{2}\right): W \times U \rightarrow Z^{*}$ is infinitely Fréchet differentiable with Lipschitz continuous first derivative, constant second derivative and vanishing third and higher derivatives. The action of the first two derivatives of $e^{1}$ are given by

$$
\begin{array}{r}
\left\langle e_{x}^{1}(x)(w, s), \phi\right\rangle=\left\langle w_{t}, \phi\right\rangle+\langle(w \cdot \nabla) y, \phi\rangle+\langle(y \cdot \nabla) w, \phi\rangle \\
+\nu(\nabla w, \nabla \phi)_{L^{2}\left(L^{2}\right)}-\langle s, \phi\rangle_{L^{2}\left(L^{2}\right)}
\end{array}
$$

where $x=(y, u) \in W \times U,(w, s) \in W \times U$ and $\phi \in L^{2}(V)$, and

$$
\begin{array}{r}
\left\langle e_{x x}^{1}(x)(w, s)(v, r), \phi\right\rangle=\left\langle e_{y y}^{1}(x)(w, v), \phi\right\rangle= \\
\langle(w \cdot \nabla) v, \phi\rangle+\langle(v \cdot \nabla) w, \phi\rangle=:\langle v, M(\phi) w\rangle_{W, W^{*}},
\end{array}
$$

where $(v, r) \in W \times U$ and $M: L^{2}(V) \rightarrow \mathcal{L}\left(W, W^{*}\right)$.

Next we introduce the Lagrange function related to problem (2.7) with $U_{\text {ad }}=U$, i.e., $\hat{L}: W \times U \times Z \rightarrow \mathbb{R}$ with

$$
\hat{L}(y, u, p)=J(y, u)+\langle p, e(y, u)\rangle_{Z, Z^{*}}
$$

According to Proposition 2.5 we have

$$
\begin{aligned}
\left\langle\hat{L}_{y y}(y, u, p) v, w\right\rangle & =\left\langle J_{y y}(y, u) v, w\right\rangle+\left\langle e_{y y}(y, u)(v, w), p\right\rangle \\
& =\langle v, w\rangle+\langle(v \cdot \nabla) w, p\rangle+\langle(w \cdot \nabla) v, p\rangle
\end{aligned}
$$

with $v, w \in W$.

3. First order optimality and the QP-subproblem. The starting point for devising algorithms to find a local solution of (1.1) are the first order necessary conditions which will be derived in this section.

According to the results in the previous section we can write (1.1) in the compact form

$$
\begin{array}{ll}
\text { minimize } & J(y, u) \quad \text { over }(y, u) \in W \times U \\
\text { subject to } & e(y, u)=0 \text { in } Z^{*} \\
& a \leq u \leq b \text { a.e. in } Q
\end{array}
$$

with $a, b \in L^{2}(Q)^{2}$.

The existence of a solution of $(\mathrm{P})$ follows from standard arguments. For the sake of completeness we include the short proof.

Theorem 3.1. Problem (P) admits a solution $\left(y^{*}, u^{*}\right) \in W \times U_{a d}$.

Proof. Let $\left\{u_{n}\right\}_{n \in \mathbb{N}} \subset U_{\text {ad }}$ be a minimizing sequence for problem (P). Due to the radial unboundedness of $J_{2}(u)$ this sequence is bounded and, thus, contains a weakly (in $U$ ) convergent subsequence, which we again denote by $\left\{u_{n}\right\}_{n \in \mathbb{N}}$. Since $U_{\text {ad }}$ is convex it is weakly closed and the limit $u^{*}$ of the subsequence is an element of $U_{\mathrm{ad}}$. The a priori estimates stated above now ensure that the unique solutions $y_{n}$ of $e\left(y_{n}, u_{n}\right)=0$ in $Z^{*}$ form a bounded sequence in $W$, which, in turn, contains a weakly (in $W$ ) convergent subsequence. Let $\tilde{y} \in W$ denote its limit. It follows from 
the analysis provided by Temam in [28] that $\tilde{y}=y\left(u^{*}\right)$. Since $J$ is weakly lower semi-continuous the pair $\left(y^{*}, u^{*}\right)$, in fact, is a solution of $(\mathrm{P})$.

In the unconstrained case, SQP-methods can be derived by applying Newton's method to the first order optimality system; see [24]. Due to the presence of the constraints on $u$ this approach has to be generalized. Associated with $(\mathrm{P})$ we consider the Lagrange functional

$$
L(x, p, \lambda)=J(x)+\langle e(x), p\rangle_{Z^{*}, Z}+\left(a-u, \lambda_{a}\right)+\left(u-b, \lambda_{b}\right),
$$

where we used $\lambda=\left(\lambda_{a}, \lambda_{b}\right) \in U \times U$ and $x=(y, u)$. Here and throughout we denote by $(\cdot, \cdot)$ the $L^{2}(Q)^{2}$ inner product. Let us next state the first order necessary conditions of $(\mathrm{P})$.

THEOREM 3.2. An optimal solution $x^{*}=\left(y^{*}, u^{*}\right) \in W \times U_{a d}$ to $(\mathrm{P})$ is characterized by the existence of Lagrange multipliers $p^{*} \in Z$ and $\left(\lambda_{a}^{*}, \lambda_{b}^{*}\right)=\lambda^{*} \in U^{2}$ satisfying

$$
\left\{\begin{array}{l}
J_{y}\left(x^{*}\right)+e_{y}^{*}(x) p^{*}=0, \\
J_{u}\left(x^{*}\right)+e_{u}^{*}\left(x^{*}\right) p^{*}-\lambda_{a}^{*}+\lambda_{b}^{*}=0, \\
e\left(x^{*}\right)=0, \\
a-u^{*} \leq 0, \quad \lambda_{a}^{*} \geq 0, \quad\left(a-u^{*}, \lambda_{a}^{*}\right)=0 \\
u^{*}-b \leq 0, \quad \lambda_{b}^{*} \geq 0, \quad\left(u^{*}-b, \lambda_{b}^{*}\right)=0 .
\end{array}\right.
$$

The last two equation in (OS) form the so-called complementarity system. Note that in the case where $U_{\mathrm{ad}}=U$ only the first three equations in (OS) with $\lambda^{*}=0$ have to be taken into account.

Given a point $(x, p, \lambda)$ close to a locally optimal solution $\left(x^{*}, p^{*}, \lambda^{*}\right)$ let us now apply a generalized Newton step to the system

$$
\left\{\begin{array}{l}
J_{y}(x)+e_{y}^{*}(x) p=0, \\
J_{u}(x)+e_{u}^{*}(x) p-\lambda_{a}+\lambda_{b}=0 \\
e(x)=0, \\
a-u \leq 0, \quad \lambda_{a} \geq 0, \quad\left(a-u, \lambda_{a}\right)=0 \\
u-b \leq 0, \quad \lambda_{b} \geq 0, \quad\left(u-b, \lambda_{b}\right)=0
\end{array}\right.
$$

To unburden the notation, subsequently we will neglect the argument $(x, p, \lambda)$. The generalized Newton step $\left(\delta_{y}, \ldots, \delta_{\lambda_{b}}\right)$ satisfies

$$
\left(\begin{array}{ccccc}
L_{y y} & 0 & e_{y}^{*} & 0 & 0 \\
0 & L_{u u} & e_{u}^{*} & -\mathrm{id} & \mathrm{id} \\
e_{y} & e_{u} & 0 & 0 & 0
\end{array}\right)\left(\begin{array}{c}
\delta_{y} \\
\delta_{u} \\
\delta_{p} \\
\delta_{\lambda_{a}} \\
\delta_{\lambda_{b}}
\end{array}\right)=-\left(\begin{array}{c}
L_{y} \\
L_{u} \\
e
\end{array}\right)
$$

for the first three equations in (OS). For the complementarity system we define

$$
\hat{\lambda}_{a}=\lambda_{a}+\delta_{\lambda_{a}}, \quad \hat{\lambda}_{b}=\lambda_{b}+\delta_{\lambda_{b}} .
$$

Thus, we obtain

$$
\begin{aligned}
& a-u-\delta_{u} \leq 0, \quad \hat{\lambda}_{a} \geq 0, \quad\left(a-u-\delta_{u}, \hat{\lambda}_{a}\right)=0 \\
& u+\delta_{u}-b \leq 0, \quad \hat{\lambda}_{b} \geq 0, \quad\left(u+\delta_{u}-b, \hat{\lambda}_{b}\right)=0
\end{aligned}
$$


Note that in the last equations in $(3.4 \mathrm{a})$ respectively $(3.4 \mathrm{~b})$ we keep the quadratic terms $-\left(\delta_{u}, \delta_{\lambda_{a}}\right)$ respectively $\left(\delta_{u}, \delta_{\lambda_{b}}\right)$. This allows us to establish a link between (3.3)(3.4) and the constrained minimization problem (QP) introduced below. Observe that the second equation in (3.3) implies

$$
L_{u u} \delta_{u}+e_{u}^{*} \delta_{p}+\hat{\lambda}=-\left(J_{u}+e_{u}^{*} p\right)
$$

where we use

$$
\hat{\lambda}=-\hat{\lambda}_{a}+\hat{\lambda}_{b}
$$

Further note that the system (3.4) can be rewritten as a nonsmooth equation of the form

$$
\max \left(\hat{\lambda}+\sigma\left(u+\delta_{u}-b\right), 0\right)+\min \left(\hat{\lambda}+\sigma\left(u+\delta_{u}-a\right), 0\right)=\hat{\lambda}
$$

for arbitrarily fixed real $\sigma>0$. In fact, it is an easy exercise to show that (3.4) and (3.6) are equivalent.

These considerations finally result in the following system which has to be solved in order to obtain the Newton direction $\left(\delta_{y}, \delta_{u}, \delta_{p}\right)$ with associated Lagrange multiplier $\hat{\lambda}$ :

$$
\left\{\begin{array}{l}
L_{y y} \delta_{y}+e_{y}^{*} \delta_{p}=-L_{y} \\
L_{u u} \delta_{u}+e_{u}^{*} \delta_{p}+\hat{\lambda}=-\left(J_{u}+e_{u}^{*} p\right) \\
e_{y} \delta_{y}+e_{u} \delta_{u}=-e \\
\Psi\left(\delta_{u}, \hat{\lambda} ; u\right)=\hat{\lambda}
\end{array}\right.
$$

with

$$
\Psi\left(\delta_{u}, \hat{\lambda} ; u\right):=\max \left(\hat{\lambda}+\sigma\left(u+\delta_{u}-b\right), 0\right)+\min \left(\hat{\lambda}+\sigma\left(u+\delta_{u}-a\right), 0\right) .
$$

In the case where the operator matrix

$$
L_{x x}:=\left(\begin{array}{cc}
L_{y y} & 0 \\
0 & L_{u u}
\end{array}\right)
$$

is positive semi-definite, the system (3.7) represents the first order necessary and sufficient condition of the QP-problem

$$
\begin{array}{ll}
\operatorname{minimize} & \left\langle\hat{L}_{x}, \delta_{x}\right\rangle+\frac{1}{2}\left\langle\hat{L}_{x x} \delta_{x}, \delta_{x}\right\rangle \quad \text { over } \delta_{x} \in W \times U \\
\text { subject to } & e+e_{x} \delta_{x}=0 \text { in } Z^{*} \\
& a-u \leq \delta_{u} \leq b-u \quad \text { a.e. in } Q
\end{array}
$$

Here we used

$$
\hat{L}(x, p)=J(x)+\langle e(x), p\rangle_{Z^{*}, Z},
$$

the Lagrangian for the unconstrained version of $(\mathrm{P})$, and $\delta_{x}=\left(\delta_{y}, \delta_{u}\right)$. Note that due to the affine linear nature of the inequality constraints we have

$$
\hat{L}_{x x}(x, p)=L_{x x}(x, p, \lambda)
$$

and further

$$
\hat{L}_{y}(x, p)=L_{y}(x, p, \lambda) .
$$

Hence, compared to the unconstrained control problem as considered, e.g., in [21] the objective functional of the QP-problems remains the same, only the additional constraints on $\delta_{u}$ must be realized. However, this requires a more sophisticated QPsolver which is the subject of section 5 . 
4. Reduced system and the SQP-algorithm. Solving, in every iteration of a numerical algorithm, a time dependent (sub)problem of the type (3.7) is a formidable task due to the size of the problem. Our aim is now to derive a reduced version of (3.7) which is more tractable numerically. For this purpose observe that the third equation in (3.7) yields

$$
\delta_{y}=-e_{y}^{-1}(x)\left(e(x)+e_{u}(x) \delta_{u}\right) .
$$

Utilizing (4.1) in the first equation of (3.7) results in

$$
\begin{aligned}
\delta_{p} & =-e_{y}^{-*}(x)\left(\hat{L}_{y}(x, p)+\hat{L}_{y y}(x, p) \delta_{y}\right) \\
& =-e_{y}^{-*}(x)\left(\hat{L}_{y}(x, p)-\hat{L}_{y y}(x, p)\left(e_{y}^{-1}(x)\left(e(x)+e_{u}(x) \delta_{u}\right)\right)\right),
\end{aligned}
$$

where $e_{y}^{-*}$ denotes the adjoint of the inverse $e_{y}^{-1}$. If we insert (4.1)-(4.2) in the second equation of (3.7), then we obtain (again after neglecting the argument $(x, p, \lambda)$ )

$$
\left(L_{u u}+e_{u}^{*} e_{y}^{-*} \hat{L}_{y y} e_{y}^{-1} e_{u}\right) \delta_{u}+\hat{\lambda}=-\hat{L}_{u}+e_{u}^{*} e_{y}^{-*}\left(\hat{L}_{y}-\hat{L}_{y y} e_{y}^{-1} e\right) .
$$

Taking into account that

$$
-\hat{L}_{u}+e_{u}^{*} e_{y}^{-*} \hat{L}_{y}=-J_{u}+e_{u}^{*} e_{y}^{-*} J_{y},
$$

then (4.3) simplifies to

$$
T^{*} \hat{L}_{x x} T \delta_{u}+\hat{\lambda}=-T^{*} J_{x}-e_{u}^{*} e_{y}^{-*} \hat{L}_{y y} e_{y}^{-1} e
$$

with

$$
T=\left(\begin{array}{c}
-e_{y}^{-1} e_{u} \\
\text { id }
\end{array}\right), \quad \text { and } \quad \hat{L}_{x x}=\left(\begin{array}{cc}
\hat{L}_{y y} & 0 \\
0 & \hat{L}_{u u}
\end{array}\right)=\left(\begin{array}{cc}
L_{y y} & 0 \\
0 & L_{u u}
\end{array}\right)
$$

Summarizing our computations, it turns out that the solution $\left(\delta_{x}, \delta_{p}, \hat{\lambda}\right)$ of $(3.7)$ can be computed by solving the reduced system

$$
\left\{\begin{array}{l}
T^{*} \hat{L}_{x x} T \delta_{u}+\hat{\lambda}=-T^{*} J_{x}-e_{u}^{*} e_{y}^{-*} \hat{L}_{y y} e_{y}^{-1} e \\
\Psi\left(\delta_{u}, \hat{\lambda} ; u\right)=\hat{\lambda}
\end{array}\right.
$$

for $\left(\delta_{u}, \hat{\lambda}\right)$ and then performing efficient backward substitution in (4.1) and (4.2).

Let $r=r(x, p)$ denote the negative right hand side in (4.4), i.e.,

$$
r=T^{*} J_{x}+e_{u}^{*} e_{y}^{-*} \hat{L}_{y y} e_{y}^{-1} e .
$$

Assuming that

$$
H=T^{*} \hat{L}_{x x} T
$$

is positive semi-definite, then the reduced system (OR) represents the first order necessary and sufficient condition for the reduced QP-problem

$$
\begin{array}{ll}
\text { minimize } & \frac{1}{2}\left\langle T^{*} \hat{L}_{x x} T \delta_{u}, \delta_{u}\right\rangle+\left\langle r, \delta_{u}\right\rangle \\
\text { subject to } & a-u \leq \delta_{u} \leq b-u \quad \text { a.e. in } Q .
\end{array}
$$


The basic SQP-algorithm will be specified next. Subsequently $\left(\mathrm{OR}^{n}\right)$ refers to problem $(\mathrm{OR})$ with $(x, p)=\left(x^{n}, p^{n}\right), n \in \mathbb{N}_{0}$.

\section{ALGORITHM 4.1. SQP-framework}

1. Choose $x^{0}=\left(y^{0}, u^{0}\right) \in W \times U_{a d}, p^{0} \in Z, \lambda^{0} \in U^{2}$, sufficiently close to a local solution; set $n=0$.

2. Do until convergence

(a) Compute the solution $\left(\delta_{u}^{n}, \hat{\lambda}^{n}\right)$ of $\left(O R^{n}\right)$.

(b) Compute $\delta_{y}^{n}, \delta_{p}^{n}$ from (4.1)-(4.2) at $\left(x^{n}, p^{n}\right)$.

(c) Update $x^{n}=x^{n-1}+\delta_{x}^{n}$ and $p^{n}=p^{n-1}+\delta_{p}^{n}$. Set $n=n+1$.

Note that the above SQP-algorithm requires globalization in order to allow an arbitrary initial choice. A globalization with respect to the requirements for unconstrained optimal control of the Navier-Stokes equations can be found in [21]. This strategy may also be applied in the present context. In fact, due to the affine character of the inequality constraints the Hessian of the constrained and the unconstrained problems coincide. Also the line search technique in [21] remains valid as long as the control iterates $u^{n}$ remain feasible during the iteration. The key ingredient of this globalization strategy is to check positive definiteness properties of

$$
H^{n}:=T^{*}\left(x^{n}\right) \hat{L}_{x x}\left(x^{n}, p^{n}\right) T\left(x^{n}\right)
$$

for all $n$. If $H^{n}$ is not positive definite in the direction $\delta_{u}^{n}$, then a positive definite approximation $\tilde{H}^{n}$ of $H^{n}$ is computed and the QP-subproblem with $H^{n}$ replaced by $\tilde{H}^{n}$ is solved yielding a new $\delta_{u}^{n}$. It can be shown that $H^{n}$ is positive definite sufficiently close to a local solution which satisfies the strong second order sufficient conditions; see [24]. This implies that we eventually have $\tilde{H}^{n}=H^{n}$. Consequently, resorting to local arguments we may assume throughout that $H^{n}$ is positive definite for all $n$.

5. An efficient QP-solver. As already noticed earlier, the computation of a solution to $\left(\mathrm{OR}^{n}\right)$ requires a more sophisticated solver compared to the unconstrained case. This is due to the nonsmooth equation $\Psi\left(\delta_{u}, \hat{\lambda} ; u\right)=\hat{\lambda}$. Here we adopt the primal-dual active set strategy (pdAS) in order to solve $\left(\mathrm{OR}^{n}\right)$.

Recall that we use $\hat{\lambda}=\lambda_{b}-\lambda_{a}$. The key step of pdAS consists in estimating the $a$-active, $b$-active and inactive sets at the solution $\left(y^{*}, u^{*}, p^{*}, \hat{\lambda}^{*}\right)$ given by

$$
\begin{array}{lc}
\mathcal{A}_{*}^{a}:=\left(\mathcal{A}_{*, 1}^{a}, \mathcal{A}_{*, 2}^{a}\right)^{\top}, \quad \mathcal{A}_{*, i}^{a}:=\left\{u_{i}^{*}=a_{i}\right\}, \quad i=1,2, \\
\mathcal{A}_{*}^{b}:=\left(\mathcal{A}_{*, 1}^{b}, \mathcal{A}_{*, 2}^{b}\right)^{\top}, \quad \mathcal{A}_{*, i}^{b}:=\left\{u_{i}^{*}=b_{i}\right\}, \quad i=1,2, \\
\mathcal{I}_{*}:=Q^{2} \backslash\left(\mathcal{A}_{*}^{a} \cup \mathcal{A}_{*}^{b}\right) \quad \text { (componentwise union), }
\end{array}
$$

respectively. Note that from our first order characterization, Theorem 3.2, we have (in the almost everywhere sense)

$$
\hat{\lambda}_{\mid \mathcal{A}_{*}^{a}}^{*} \leq 0, \quad \hat{\lambda}_{\mid \mathcal{A}_{*}^{b}}^{*} \geq 0, \quad \hat{\lambda}_{\mid \mathcal{I}_{*}}^{*}=0
$$

Now assume that we are given $u^{n}$ and we want to determine the solution $\delta_{u}^{n}, \hat{\lambda}^{n}$ to $\left(\mathrm{OR}^{n}\right)$ by the pdAS. In view of $(5.1)$ and (5.2), the aim is to compute $\left(\delta_{u}^{n}, \hat{\lambda}^{n}\right)$ such that $u^{n}+\delta_{u}^{n}$ and $\hat{\lambda}^{n}$ satisfy

$$
a \leq u^{n}+\delta_{u}^{n} \leq b \quad \text { and } \quad \hat{\lambda}_{\mid \mathcal{A}_{n}^{a}}^{n} \leq 0, \quad \hat{\lambda}_{\mid \mathcal{A}_{n}^{b}}^{n} \geq 0, \quad \hat{\lambda}_{\mid \mathcal{I}_{n}}^{n}=0
$$


simultaneously. Given estimates $\delta_{u, l-1}$ and $\hat{\lambda}_{l-1}$ of $\delta_{u}^{n}$ and $\hat{\lambda}^{n}$, we define the following approximations of the active and inactive sets in $(5.1)$ by $(i=1,2)$

$$
\begin{array}{ll}
\mathcal{A}_{l}^{a}:=\left(\mathcal{A}_{l, 1}^{a}, \mathcal{A}_{l, 2}^{a}\right)^{\top}, & \mathcal{A}_{l, i}^{a}:=\left\{\left(\hat{\lambda}_{l-1}+\sigma\left(u^{n}+\delta_{u, l-1}-a\right)\right)_{i}<0\right\}, \\
\mathcal{A}_{l}^{b}:=\left(\mathcal{A}_{l, 1}^{b}, \mathcal{A}_{l, 2}^{b}\right)^{\top}, \quad \mathcal{A}_{l, i}^{b}:=\left\{\left(\hat{\lambda}_{l-1}+\sigma\left(u^{n}+\delta_{u, l-1}-b\right)\right)_{i}>0\right\}, \\
\mathcal{I}_{l}:=Q^{2} \backslash\left(\mathcal{A}_{l}^{a} \cup \mathcal{A}_{l}^{b}\right) \quad \text { (componentwise union). }
\end{array}
$$

Above the scalar $\sigma>0$ is arbitrarily fixed. In section 5.1 we will see that $\sigma=\alpha>0$ is of particular interest. The choice (5.3) is related to (3.6). In the discussion on p. 13 below a detailed motivation is given. As it will turn out, it is associated to a generalized derivative of $\Psi$.

Let us next specify the pdAS as utilized in step (2a) of the SQP-algorithm. For convenience we use $r^{n}=r\left(x^{n}, p^{n}\right)$.

\section{Algorithm 5.1. Primal-dual active set strategy.}

(2a.0) Initialize $\delta_{u, 0}=0, \hat{\lambda}_{0}=-r^{n}$; set $l=1$. Choose a small $\epsilon>0$.

(2a.1) Determine $\mathcal{A}_{l}^{a}, \mathcal{A}_{l}^{b}$ and $\mathcal{I}_{l}$ from (5.3).

(2a.2) If $l \geq 2$ and $\mathcal{A}_{l}^{a}=\mathcal{A}_{l-1}^{a}, \mathcal{A}_{l}^{b}=\mathcal{A}_{l-1}^{b}$, or

$$
\left|\Psi\left(\delta_{u, l-1}, \hat{\lambda}_{l-1} ; u^{n}\right)-\hat{\lambda}_{l-1}\right|_{\left(L^{2}\right)^{2}} \leq \epsilon,
$$

then $\delta_{u}^{n}=\delta_{u, l-1}, \hat{\lambda}^{n}=\hat{\lambda}_{l-1}$ and RETURN; otherwise go to step (2a.3).

(2a.3) Fix

$$
\begin{aligned}
\delta_{u, l} & =a-u^{n} \quad \text { on } \mathcal{A}_{l}^{a}, \\
\delta_{u, l} & =b-u^{n} \quad \text { on } \mathcal{A}_{l}^{b}, \\
\hat{\lambda}_{l} & =0 \quad \text { on } \mathcal{I}_{l}
\end{aligned}
$$

and obtain $\left.\delta_{u, l}\right|_{\mathcal{I}_{l}},\left.\hat{\lambda}_{l}\right|_{\mathcal{A}_{l}^{a} \cup \mathcal{A}_{l}^{b}}$ from solving

$$
H^{n} \delta_{u, l}+\hat{\lambda}_{l}=-r^{n}
$$

Put $l:=l+1$ and go to (2a.1).

The reduced problem (5.4), which has to be solved in every iteration of the pdAS, can hardly be solved by means of direct solvers due to the size of the problem. Since $H^{n}$ is positive definite and symmetric, we apply the conjugate gradient (CG) method. In the context of pdAS the CG-method has to take care of the particular settings according to step (2a.3) of pdAS, i.e.,

$$
\delta_{u, l \mid \mathcal{A}_{l}^{a}}=\left(a-u^{n}\right)_{\mid \mathcal{A}_{l}^{a}}, \quad \delta_{u, l \mid \mathcal{A}_{l}^{b}}=\left(b-u^{n}\right)_{\mid \mathcal{A}_{l}^{b}}, \quad \text { and } \quad \hat{\lambda}_{l \mid \mathcal{I}_{l}}=0 .
$$

Hence, the CG-method operates essentially only on $\mathcal{I}_{l}$. Consequently, we consider the following subspace CG-method where $\mathcal{A}_{l}=\mathcal{A}_{l}^{a} \cup \mathcal{A}_{l}^{b}$.

\section{Algorithm 5.2. Subspace CG-method.}

(2a.3.0) Initialize

$$
\begin{aligned}
& v_{0 \mid \mathcal{I}_{l}}:=0, \quad v_{0 \mid \mathcal{A}_{l}^{a}}=\left(a-u^{n}\right)_{\mid \mathcal{A}_{l}^{a}}, \quad v_{0 \mid \mathcal{A}_{l}^{b}}=\left(b-u^{n}\right)_{\mid \mathcal{A}_{l}^{b}}, \\
& d_{0 \mid \mathcal{I}_{l}}=r_{\mid \mathcal{I}_{l}}^{n}-\left(H^{n} v_{0}\right)_{\mid \mathcal{I}_{l}}=: g_{0 \mid \mathcal{I}_{l}}, \quad k:=0 .
\end{aligned}
$$


(2a.3.1) Do until convergence

$$
\begin{aligned}
& \text { (a) } t_{k}:=\frac{\left|g_{k \mid I_{l}}\right|_{\left(L^{2}\right)^{2}}^{2}}{\left(d_{k}, H^{n} d_{k}\right)}, \\
& \text { (b) } v_{k+1}=v_{k}+t_{k} d_{k}, \\
& \text { (c) } g_{k+1 \mid \mathcal{I}_{l}}=g_{k \mid \mathcal{I}_{l}}+t_{k}\left(H^{n} d_{k}\right)_{\mid \mathcal{I}_{l}}, \\
& \text { (d) } \beta_{k}=\frac{\left|g_{k+1 \mid \mathcal{I}_{l}}\right|_{\left(L^{2}\right)^{2}}}{\left|g_{k \mid I_{l}}\right|_{\left(L^{2}\right)^{2}}^{2}}, \\
& \text { (e) } d_{k+1 \mid \mathcal{I}_{l}}=-g_{k+1 \mid \mathcal{I}_{l}}+\beta_{k} d_{k \mid \mathcal{I}_{l}}, \\
& \text { (f) } d_{k+1 \mid \mathcal{A}_{l}}=0, \\
& \text { (g) } k=k+1 .
\end{aligned}
$$

(2a.3.2) Set $\delta_{u, l}=v_{k}$ and compute $\hat{\lambda}_{l}=-r^{n}-H^{n} \delta_{u, l}$.

Note that we do not require a partitioning of $H^{n}$ according to active and inactive sets. Rather we achieve the settings (5.5) by fixing $d_{k+1 \mid \mathcal{A}_{l}}=0$ in step (2a.3.1.f). Finally, we remark that the stopping tolerance $\epsilon>0$ of the pdAS and the criterion for terminating the subspace CG-method have to be adjusted appropriately; see our choices in section 7 .

5.1. Convergence properties of the primal-dual active set strategy. Let us now turn towards the convergence analysis of the primal-dual active set strategy. For this purpose we recall the concept of slant differentiability of a function as introduced in [7]. In [22] this concept is utilized for proving locally superlinear convergence of the primal dual active set strategy for a class of constrained optimization problems in function spaces. This convergence result relies on the fact that the primal-dual active set strategy is equivalent to a semismooth Newton method.

Let $F: D \subset X \rightarrow Y$ be a mapping from an open subset $D$ of the Banach space $X$ with values in the Banach space $Y$. The following definition is taken from [7] (see also [22]).

Definition 5.3. The mapping $F: D \subset X \rightarrow Y$ is called slantly differentiable in $D$ if there exists a family of mappings $G \in \mathcal{L}(X, Y)$ satisfying

$$
\lim _{h \rightarrow 0} \frac{\|F(x+h)-F(x)-G(x+h) h\|_{Y}}{\|h\|_{X}}=0 \quad \text { for } x \in D .
$$

The mapping $G$ is called slanting function for $F$ in $D$.

In [22] (see also [32]) it is observed that $\max : L^{q_{1}}(\Omega) \rightarrow L^{q_{2}}(\Omega)$ is slantly differentiable for $1 \leq q_{2}<q_{1} \leq+\infty$. If $q_{1} \leq q_{2}$ this property does not hold true. Note that in our problem setting we have $u, \delta_{u}, \hat{\lambda} \in L^{2}(Q)^{2}$. Hence, the slant differentiability concept with respect to $\delta_{u}$ or $\hat{\lambda}$ cannot be applied to $\Psi\left(\delta_{u}, \hat{\lambda} ; u\right)$ immediately.

In our numerical tests we use the following modified version of the primal dual active set algorithm which operates on $\delta_{u}$ only. For its derivation consider equation (4.3) which gives a relation between $\hat{\lambda}$ and $\delta_{u}$, i.e.,

$$
\hat{\lambda}=-\hat{L}_{u}+e_{u}^{*} e_{y}^{-*}\left(\hat{L}_{y}-\hat{L}_{y y} e_{y}^{-1}\left(e+e_{u} \delta_{u}\right)\right)-L_{u u} \delta_{u} .
$$

Note that the cost functionals in (2.4) and (2.5) yield

$$
J_{u}(u)=\alpha u, \quad \text { and } \quad L_{u u}(x, p, \lambda) \delta_{u}=\alpha \delta_{u} .
$$

Thus, from (5.6) it follows

$$
\hat{\lambda}=-\alpha\left(u+\delta_{u}\right)+S \delta_{u}+t
$$


with

$$
\begin{aligned}
t=t(y, u, p) & =e_{u}^{*} e_{y}^{-*}\left(J_{y}-\hat{L}_{y y} e_{y}^{-1} e\right), \\
S=S(y, u, p) & =-e_{u}^{*} e_{y}^{-*} \hat{L}_{y y} e_{y}^{-1} e_{u} .
\end{aligned}
$$

Using (5.7) for $\hat{\lambda}$ in $\Psi\left(\delta_{u}, \hat{\lambda} ; u\right)$ with the particular choice $\sigma=\alpha$ yields

$$
\hat{\Psi}\left(\delta_{u}\right):=\max \left(S \delta_{u}-\alpha b+t, 0\right)+\min \left(S \delta_{u}-\alpha a+t, 0\right) .
$$

Relation (5.8) allows us to replace the componentwise active set estimates in (5.3) by

$$
\begin{array}{ll}
\mathcal{A}_{l, i}^{a}:=\left\{\left(S^{n} \delta_{u, l-1}-\alpha a+t^{n}\right)_{i}<0\right\}, & i=1,2, \\
\mathcal{A}_{l, i}^{b}:=\left\{\left(S^{n} \delta_{u, l-1}-\alpha b+t^{n}\right)_{i}>0\right\}, & i=1,2 .
\end{array}
$$

Here we use $S^{n}=S\left(y^{n}, u^{n}, p^{n}\right)$ and analogously for $t^{n}$. We call the resulting algorithm, which iterates on $\delta_{u, l}$ only, the reduced primal-dual active set strategy (rpdAS).

As a candidate for a slanting function for $\hat{\Psi}$ we consider

$$
G\left(\delta_{u}\right)=g_{\max }\left(S \delta_{u}-\alpha b+t\right) S+g_{\min }\left(S \delta_{u}-\alpha a+t\right) S,
$$

with

$$
g_{\max }(w)(x)= \begin{cases}1 & \text { if } w(x)>0 \\ 0 & \text { else }\end{cases}
$$

and

$$
g_{\min }(w)(x)= \begin{cases}1 & \text { if } w(x)<0 \\ 0 & \text { else }\end{cases}
$$

Let us motivate this particular choice of $G$ with respect to the pdAS, respectively rpdAS. Assume for the moment that $G$ is a slanting function for $\hat{\Psi}$. We apply a generalized version of Newton's method for iteratively solving

$$
\begin{aligned}
H^{n} \delta_{u}+\hat{\lambda}+r^{n} & =0, \\
\hat{\Psi}\left(\delta_{u}\right)+\alpha\left(u+\delta_{u}\right)-S \delta_{u}-t & =0 .
\end{aligned}
$$

Suppose that $\delta_{u, l-1}, \hat{\lambda}_{l-1}$ are the actual iterates in the Newton process. Then, using $G\left(\delta_{u, l-1}\right)$, we obtain the following equations for the increments $d_{u}, d_{\hat{\lambda}}$.

$$
\begin{gathered}
H^{n} d_{u}+d_{\hat{\lambda}}=-r^{n}-H^{n} \delta_{u, l-1}-\hat{\lambda}_{l-1}, \\
\chi_{\mathcal{A}_{l}^{b}} S d_{u}+\chi_{\mathcal{A}_{l}^{a}} S d_{u}+\alpha d_{u}-S d_{u}=-\hat{\Psi}\left(\delta_{u, l-1}\right)-\alpha\left(u+\delta_{u}\right)+S \delta_{u}+t .
\end{gathered}
$$

By $\chi_{\mathcal{S}}$ we denote the characteristic function of a set $\mathcal{S} \subset Q^{2}$. On $\mathcal{A}_{l}^{b}$ equation $(5.12 \mathrm{~b})$ yields

$$
\delta_{u, l-1}+d_{u}=b-u
$$

Analogously, on $\mathcal{A}_{l}^{a}$ we obtain

$$
\delta_{u, l-1}+d_{u}=a-u
$$


Thus rpdAS is regained. As a consequence, if $G$ in (5.10) is a slanting function for $\hat{\Psi}$, then rpdAS is equivalent to a generalized Newton method for the non-differentiable system (5.11).

With respect to the desired slant differentiability relation we have the following result.

Proposition 5.4. Let Assumption 2.2 be satisfied with $\epsilon \in\left(0, \frac{1}{3}\right]$ and let $\delta_{\epsilon}$ as in Proposition 2.1. Further let $q \in\left(2, \delta_{\epsilon}\right)$ be arbitrarily fixed, and $a, b \in L^{q}(Q)^{2}$. Then the mapping $G$ defined in (5.10) is a slanting function for $\hat{\Psi}: L^{2}(Q)^{2} \rightarrow L^{2}(Q)^{2}$ with $t \in L^{q}(Q)^{2}, y \in W, p \in Z$ and $S: L^{2}(Q)^{2} \rightarrow W_{1+\epsilon}^{2} \hookrightarrow L^{q}(Q)^{2}$.

Proof. First observe that $e_{u}=(-\mathrm{id}, 0)$ which, by Proposition 2.3, yields $e_{y}^{-1} e_{u} \delta_{u} \in$ $W$ for $\delta_{u} \in L^{2}(Q)^{2}$. Next consider $w=\hat{L}_{y y} v$ with $v=e_{y}^{-1} e_{u} \delta_{u}$ in detail. Since $p \in Z$ and $y \in W$ a straightforward estimation gives $\left\langle e_{y y}(y, u)(\cdot, v), p\right\rangle_{Z^{*}, Z} \in L^{\frac{4}{3}}\left(V^{*}\right) \cap W^{*}$. Since by Assumption $2.2 J_{1_{y y}}(y) v \in L^{1+\epsilon}\left(V^{*}\right) \cap W^{*}$ we obtain $\hat{L}_{y y} v \in L^{1+\epsilon}\left(V^{*}\right) \cap W^{*}$. The regularity results of Proposition 2.4 now yield

$$
z=e_{y}^{-*} w \in W_{1+\epsilon}^{2}
$$

where by Proposition 2.1 the space $W_{1+\epsilon}^{2}$ continuously embeds into $L^{p}(Q)^{2}$ for all $2<p<\delta_{\epsilon}$. Since $e_{u}=(-\mathrm{id}, 0)$, this immediately yields $S \delta_{u} \in L^{q}(Q)^{2}$. A similar argument proves that $e_{y}^{-*} J_{y}$ and $e_{y}^{-*} \hat{L}_{y y} e_{y}^{-1} e$ are elements of $W_{1+\epsilon}^{2}$, respectively, and hence $t \in L^{q}(Q)^{2}$.

For the remainder of the assertion we restrict ourselves to proving that $\hat{\psi}$ : $L^{2}(Q)^{2} \rightarrow L^{2}(Q)^{2}$, with

$$
\hat{\psi}\left(\delta_{u}\right)=\max \left(S \delta_{u}-\alpha b+t, 0\right)
$$

is slantly differentiable with slanting function

$$
G_{\max }\left(\delta_{u}\right)=g_{\max }\left(S \delta_{u}-\alpha b+t, 0\right) S .
$$

Applying the analogous arguments to the min-term in $\hat{\Psi}$ then proves slant differentiability of $\hat{\Psi}$ with the slanting function $G$ as defined in (5.10).

For $h \in L^{2}(Q)^{2}$ and $2<q<\delta_{\epsilon}$ consider

$$
\begin{aligned}
& \lim _{h \rightarrow 0} \frac{\left|\hat{\psi}\left(\delta_{u}+h\right)-\hat{\psi}\left(\delta_{u}\right)-G_{\max }\left(\delta_{u}+h\right) h\right|_{\left(L^{2}\right)^{2}}}{|h|_{\left(L^{2}\right)^{2}}}= \\
& \lim _{h \rightarrow 0} \frac{|S h|_{\left(L^{q}\right)^{2}}}{|h|_{\left(L^{2}\right)^{2}}} \frac{\left|\hat{\psi}\left(\delta_{u}+h\right)-\hat{\psi}\left(\delta_{u}\right)-G_{\max }\left(\delta_{u}+h\right) h\right|_{\left(L^{2}\right)^{2}}}{|S h|_{\left(L^{q}\right)^{2}}}=
\end{aligned}
$$

Since $S: L^{2}(Q)^{2} \rightarrow L^{q}(Q)^{2}$ is bounded we may proceed as in the proof of [22, Theorem 4.1] to argue

$$
\begin{aligned}
& \lim _{h \rightarrow 0} \frac{|S h|_{\left(L^{q}\right)^{2}}}{|h|_{\left(L^{2}\right)^{2}}} \frac{\left|\hat{\psi}\left(\delta_{u}+h\right)-\hat{\psi}\left(\delta_{u}\right)-G_{\max }\left(\delta_{u}+h\right) h\right|_{\left(L^{2}\right)^{2}}}{|S h|_{\left(L^{q}\right)^{2}}} \leq \\
& C \lim _{h \rightarrow 0} \frac{\left|\hat{\psi}\left(\delta_{u}+h\right)-\hat{\psi}\left(\delta_{u}\right)-G_{\max }\left(\delta_{u}+h\right) h\right|_{\left(L^{2}\right)^{2}}}{|S h|_{\left(L^{q}\right)^{2}}}=0
\end{aligned}
$$

with some constant $C>0$. This completes the proof. 
Before we state our convergence result for the rpdAS let us discuss the assumptions in Proposition 5.4. We define $\tilde{Z}=W_{1+\epsilon}^{2} \times H$, and we suppose that $a, b \in L^{q}(Q)^{2}$ with $q>2$ are given. Assume further that the SQP-method is initialized by

$$
\left(y^{0}, u^{0}, p^{0}, \hat{\lambda}^{0}\right) \in W \times L^{2}(Q)^{2} \times \tilde{Z} \times L^{2}(Q)^{2} .
$$

Note that by the structure of the QP-problems (QP) at $(y, u, p)=\left(y^{n-1}, u^{n-1}, p^{n-1}\right)$, $n \geq 1$, the corresponding first order system analogous to (3.7), and Propositions 2.3 and 2.4 we have

$$
\left(\delta_{y}^{n}, \delta_{u}^{n}, \delta_{p}^{n}, \hat{\lambda}^{n}\right) \in W \times L^{2}(Q)^{2} \times \tilde{Z} \times L^{2}(Q)^{2} .
$$

Consequently, the iterates of the SQP-algorithm satisfy

$$
\left(y^{n}, u^{n}, p^{n}, \hat{\lambda}^{n}\right) \in W \times L^{2}(Q)^{2} \times \tilde{Z} \times L^{2}(Q)^{2} \quad \text { for all } n \geq 0 .
$$

Applying Proposition 5.4 yields for all $n \in \mathbb{N}_{0}$

$$
t^{n}=t\left(y^{n}, u^{n}, p^{n}\right) \in L^{q}(Q)^{2} \quad \text { for all } 2<q<\delta_{\epsilon} .
$$

Now we can apply the convergence result of [7] (see also [22, 32]) for Newton's method for slantly differentiable mappings.

TheOREM 5.5. Let the assumptions of Proposition 5.4 be satisfied. Then the reduced pdAS is equivalent to a generalized Newton method and converges at a locally superlinear rate, i.e.,

$$
\left|\delta_{u, l+1}-\delta_{u}^{n}\right|_{\left(L^{2}\right)^{2}}=\mathcal{O}\left(\left|\delta_{u, l}-\delta_{u}^{n}\right|_{\left(L^{2}\right)^{2}}\right) \quad \text { for all } l,
$$

with $\delta_{u, 0}$ sufficiently close to $\delta_{u}^{n}$, the solution to $\left(O R^{n}\right)$.

The above convergence result is only a local result, i.e., one has to find an initial point close to the solution of the QP-subproblem (OR). In our numerical test runs we have never observed problems with respect to convergence of the rpdAS with initialization $\delta_{u, 0}=0$. In fact, after 2-4 SQP iterations it turns out that the rpdAS requires only one iteration to terminate successfully; see, e.g., Tables 7.1-7.4 in section 7. Further, if the SQP-method converges to a solution (stationary point) of the control problem, we have that $\delta_{u}^{n}$ approaches zero in the course of the SQP-iterations. Thus, it is to expect that initializing with $\delta_{u, 0}=0$ yields a starting point for rpdAS such that Theorem 5.5 holds true.

6. Convergence analysis of the SQP-iteration. We analyze the SQP-iteration by utilizing generalized equations; see, e.g., [27]. Let $\epsilon \in\left(0, \frac{1}{3}\right]$, and let $2<q<\delta_{\epsilon}$ with $\delta_{\epsilon}$ as is Proposition 2.1. We consider the spaces

$$
\begin{aligned}
& D=W \times L^{q}(Q)^{2} \times \tilde{Z}, \\
& R=L^{1+\epsilon}\left(V^{*}\right) \cap W^{*} \times Z^{*} \times L^{q}(Q)^{2},
\end{aligned}
$$

and we recall the definitions of $Z, W$ and $W_{1+\epsilon}^{2}$ in (2.1)-(2.2). The norms $|\cdot|_{D}$ and $|\cdot|_{R}$ are the sums of the component norms, respectively. From now on we assume that $a, b \in L^{q}(Q)^{2}$. Note that we use $L^{q}(Q)^{2}$ in the definitions of $D$ and $R$ rather than $L^{2}(Q)^{2}$. This is feasible because if we initialize the SQP-method by $u^{0} \in L^{q}(Q)^{2}$ and observe that due to $a, b \in L^{q}(Q)^{2}$ we have $\delta_{u}^{n} \in L^{q}(Q)^{2}$ for all $n$, then $u^{n}+\delta_{u}^{n} \in L^{q}(Q)^{2}$ for all $n$. Utilizing the tools employed in the proof of Proposition 5.4 for the analysis of $r^{n}$ and $H^{n} \delta_{u}^{n}$, we further obtain $\hat{\lambda}^{n} \in L^{q}(Q)^{2}$ for all $n$. 
First we convert the system (3.2) into the generalized equation

$$
0 \in F(d)+T(u),
$$

with $d=(y, u, p)$, by defining $F: D \rightarrow R$ as

$$
\begin{aligned}
& F_{1}(d):=J_{y}(y, u)+e_{y}^{*}(y, u) p, \\
& F_{2}(d):=e(y, u), \\
& F_{3}(d):=J_{u}(y, u)+e_{u}^{*}(y, u) p,
\end{aligned}
$$

and the set-valued map $T: D \rightarrow 2^{R}$ as

$$
T(u):=(\{0\},\{0\}, N(u)) .
$$

By $N(u)$ we denote the cone

$$
N(u)= \begin{cases}\left\{\varphi \in L^{q}(Q)^{2}:(\varphi, v-u) \leq 0 \text { for all } v \in U_{\mathrm{ad}}\right\}, & \text { if } u \in U_{\mathrm{ad}}, \\ \emptyset, & \text { else. }\end{cases}
$$

Observe that $N(u)$ is the normal cone of $U_{\text {ad }} \subset U$ intersected with $L^{q}(Q)^{2}$. Further $T$ has a closed graph, and $F$ is of class $\mathcal{C}^{1,1}$.

The generalized Newton method for (6.1) is defined as follows. Let $d^{n}$ denote the actual iterate. Then the next iterate $d^{n+1}$ is defined by

$$
\text { find } \begin{aligned}
\delta_{d}: & 0 \in F\left(d^{n}\right)+F^{\prime}\left(d^{n}\right) \delta_{d}+T\left(d^{n}+\delta_{d}\right), \\
& d^{n+1}=d^{n}+\delta_{d} .
\end{aligned}
$$

A straightforward computation verifies that $(6.2)$ is equivalent to $(3.7)$ at $(y, u, p)=$ $\left(y^{n}, u^{n}, p^{n}\right)$.

In order to prove quadratic convergence of the process (6.2) we use the concept of strong regularity of (6.1). The notion of strong regularity of a generalized equation was introduced in [27].

Definition 6.1. The inclusion (6.1) is called strongly regular at $d^{*} \in D$ if there exist $r_{1}, r_{2}, C_{L}>0$ such that for all perturbations $\eta \in B_{r_{1}}\left(0_{R}\right)$ the linearized equation

$$
\eta \in F\left(d^{*}\right)+F^{\prime}\left(d^{*}\right)\left(d-d^{*}\right)+T(d)
$$

admits a unique solution $d \in B_{r_{2}}\left(d^{*}\right)$ with a Lipschitz continuous solution operator $d: B_{r_{1}}\left(0_{R}\right) \rightarrow B_{r_{2}}\left(d^{*}\right)$, i.e.,

$$
\left|d\left(\eta_{1}\right)-d\left(\eta_{2}\right)\right|_{D} \leq C_{L}\left|\eta_{1}-\eta_{2}\right|_{R} \text { for all } \eta_{1}, \eta_{2} \in B_{r_{1}}\left(0_{R}\right) .
$$

For the following discussion we rely on the assumption which is stated next. Let $x^{*}=\left(y^{*}, u^{*}\right)$ denote a solution to problem (1.1) with $p^{*}$ as the corresponding adjoint state.

Assumption 6.2.

- There exists $c>0$ such that $\left(J_{u u}\left(x^{*}\right) v, v\right)_{U} \geq c|v|_{U}^{2}$ for all $v \in U$.

- $J_{y y}\left(x^{*}\right)$ is positive semi-definite.

- $J_{y}\left(x^{*}\right) \in L^{1+\epsilon} \cap W^{*}$ is sufficiently small.

We are now prepared to formulate the local convergence theorem for (6.2).

TheOREM 6.3. Let $d^{*}$ denote a solution of (6.1) which satisfies Assumption 6.2. Then there exist constants $r>0$ and $C>0$ such that for all starting values $d^{0} \in$ 
$B_{r}\left(d^{*}\right)$ the generalized Newton method (6.2) generates a sequence $\left\{d^{n}\right\} \subset B_{r}\left(d^{*}\right)$ which converges quadratically to $d^{*}$, i.e.,

$$
\left|d^{n+1}-d^{*}\right|_{D} \leq C\left|d^{n}-d^{*}\right|_{D}^{2} \text { for all } n \geq 0 .
$$

Following the concepts in $[1,12,30]$, for a proof of Theorem 6.3 we first investigate the strong regularity of $(6.1)$ at $d^{*}=\left(y^{*}, u^{*}, p^{*}\right)$. This is the contents of the next lemma.

Lemma 6.4. Let $d^{*}$ denote a solution of (6.1) which satisfies Assumption 6.2. Then the generalized equation (6.1) is strongly regular at $d^{*}$.

Proof. We check the conditions of definition 6.1 by utilizing the analysis developed in [24].

First we show that the generalized equation (6.3) admits a unique solution $d=$ $(x, p)^{\top}$. For this purpose let $\eta=\left(\eta_{1}, \eta_{2}, \eta_{3}\right)^{\top} \in R$, and define

$$
\begin{array}{r}
f(y, u):=\left\langle J_{y}, \delta_{y}\right\rangle_{W^{*}, W}+\frac{1}{2}\left\langle J_{y y} \delta_{y}, \delta_{y}\right\rangle_{W^{*}, W}+\left\langle e_{y y}\left(\delta_{y}, \delta_{y}\right), p^{*}\right\rangle_{Z^{*}, Z}+\left(J_{u}, \delta_{u}\right)_{U} \\
+\frac{1}{2}\left(J_{u u}\left(\delta_{u}\right), \delta_{u}\right)_{U}-\left\langle\eta_{1}, \delta_{y}\right\rangle_{W^{*}, W}-\left(\eta_{3}, \delta_{u}\right)_{U}
\end{array}
$$

where we use $\delta_{y}=y-y^{*}, \delta_{u}=u-u^{*}, J=J\left(x^{*}\right)$ and $e=e\left(x^{*}\right)$ for the ease of notation. Next we consider the minimization problem

$$
\min _{y, u} f(y, u) \text { s.t. } e+e_{y} \delta_{y}+e_{u} \delta_{u}=\eta_{2} \text { in } Z^{*} \text { and } u \in U_{\mathrm{ad}} .
$$

Note that its necessary optimality condition coincides with (6.3). These conditions also would be sufficient if $f$ would be convex. But lack of convexity can only arise through the term

$$
\left\langle e_{y y}\left(\delta_{y}, \delta_{y}\right), p^{*}\right\rangle_{Z^{*}, Z}
$$

since it may be negative. Using a similar technique to the one in the proof of [24, Lemma 5.1], it is not difficult to show that, for $J_{y}\left(x^{*}\right)$ small enough, there holds for some $\kappa>0$

$$
\ell_{x x}(d)\left(\delta_{x}, \delta_{x}\right) \geq \kappa\left(\left|\delta_{y}\right|_{W}^{2}+\left|\delta_{u}\right|_{L^{2}(Q)^{2}}^{2}\right)
$$

for all $\delta_{x} \in W \times U_{\text {ad }}$ with $e_{y} \delta_{y}+e_{u} \delta_{u}=0$ in $Z^{*}$. Here we have set $\delta_{x}=\left(\delta_{y}, \delta_{u}\right)$, and $\ell$ denotes the Lagrangian associated to the minimization problem (6.4), i.e., $\ell: W \times U_{\text {ad }} \times \tilde{Z}$ with

$$
\ell(d)=f(y, u)+\left\langle e+e_{y}\left(y-y^{*}\right)+e_{u}\left(u-u^{*}\right)-\eta_{2}, p\right\rangle_{Z^{*}, Z}
$$

The relation (6.5) implies that (6.4), under Assumption 6.2, admits a unique solution $(y(\eta), u(\eta))$.

Next we argue Lipschitz continuity of the solution w.r.t $\eta$. First note that the solution $(y(\eta), u(\eta))$ satisfies the following variational inequality:

$\ell^{\prime}(y(\eta), u(\eta), p(\eta))(y-y(\eta), u-u(\eta), p-p(\eta)) \geq 0 \quad$ for all $(y, u, p) \in W \times U_{\text {ad }} \times Z$. 
Here $p(\eta)$ denotes the adjoint state associated with $(y(\eta), u(\eta))$. We denote by prime the differentiation w.r.t. $(y, u, p)$, and we use $d(\eta)=(y(\eta), u(\eta), p(\eta))$. Now let $\eta^{1}, \eta^{2} \in R$ be given. To simplify the notion we define $\delta_{y}^{\eta}=y\left(\eta^{1}\right)-y\left(\eta^{2}\right)$ and analogously for $\delta_{u}^{\eta}, \delta_{x}^{\eta}$, and $\delta_{p}^{\eta}$. Below the constant $C$ can take different values on different occasions. A straightforward computation shows that

$$
\begin{aligned}
0 \leq & \ell^{\prime}\left(d\left(\eta^{2}\right)\right)\left(\delta_{x}^{\eta}, \delta_{p}^{\eta}\right)-\ell^{\prime}\left(d\left(\eta^{1}\right)\right)\left(\delta_{x}^{\eta}, \delta_{p}^{\eta}\right) \\
= & \left\langle\eta_{1}^{1}-\eta_{1}^{2}, \delta_{y}^{\eta}\right\rangle_{W^{*}, W}-\left\langle\eta_{2}^{1}-\eta_{2}^{2}, \delta_{p}^{\eta}\right\rangle_{Z^{*}, Z}+\left(\eta_{3}^{1}-\eta_{3}^{2}, \delta_{u}^{\eta}\right) \\
& -\ell^{\prime \prime}\left(d^{*}\right)\left(\delta_{x}^{\eta}, \delta_{x}^{\eta}\right) .
\end{aligned}
$$

Proposition 2.3 and 2.4 yield

$$
\begin{aligned}
\left|\delta_{y}^{\eta}\right|_{W} & \leq C\left(\left|\delta_{u}^{\eta}\right|_{L^{2}(Q)^{2}}+\left|\eta_{2}^{1}-\eta_{2}^{2}\right|_{Z^{*}}\right), \\
\left|\delta_{p}^{\eta}\right|_{Z} & \leq C\left(\left|\delta_{y}^{\eta}\right|_{W}+\left|\eta_{1}^{1}-\eta_{1}^{2}\right|_{W^{*}}\right) .
\end{aligned}
$$

¿From (6.5) and (6.7)-(6.9) we infer

$$
\kappa\left|\delta_{u}^{\eta}\right|_{L^{2}(Q)^{2}}^{2} \leq C\left(\left|\eta^{1}-\eta^{2}\right|_{R}\left|\delta_{u}^{\eta}\right|_{L^{2}(Q)^{2}}+\left|\eta^{1}-\eta^{2}\right|_{R}^{2}\right) .
$$

Using Young's inequality we obtain

$$
\left|\eta^{1}-\eta^{2}\right|_{R}\left|\delta_{u}^{\eta}\right|_{L^{2}(Q)^{2}} \leq \frac{1}{2 \kappa}\left|\eta^{1}-\eta^{2}\right|_{R}^{2}+\frac{\kappa}{2}\left|\delta_{u}^{\eta}\right|_{L^{2}(Q)^{2}}^{2} .
$$

Therefore (6.10) implies

$$
\left|\delta_{u}^{\eta}\right|_{L^{2}(Q)^{2}} \leq C\left|\eta^{1}-\eta^{2}\right|_{R}
$$

Further Proposition 2.4 yields the existence of a constant $C>0$ such that

$$
\left|\delta_{p}^{\eta}\right|_{\tilde{Z}} \leq C\left(\left|\delta_{y}^{\eta}\right|_{W}+\left|\eta_{1}^{1}-\eta_{1}^{2}\right|_{L^{1+\epsilon}\left(V^{*}\right) \cap W^{*}}\right)
$$

The variational inequality (6.6) yields

$$
\alpha u(\eta)-\eta_{3}+e_{u}^{*} p(\eta) \in N(u(\eta)) .
$$

This is equivalent to

$$
u(\eta)=P_{U_{\text {ad }}}\left(\alpha^{-1}\left(\eta_{3}-e_{u}^{*} p(\eta)\right)\right) \in L^{q}(Q)^{2},
$$

where $P_{U_{\text {ad }}}$ denotes the (pointwise) projection onto $U_{\text {ad }}$. Hence, we have

$$
\left|u\left(\eta^{1}\right)-u\left(\eta^{2}\right)\right|_{L^{q}(Q)^{2}} \leq C\left(\left|\eta_{3}^{1}-\eta_{3}^{2}\right|_{L^{q}(Q)^{2}}+\left|\delta_{p}^{\eta}\right|_{\tilde{Z}}\right) .
$$

Finally, combining the last estimate with (6.11)-(6.12) and (6.8) results in

$$
\left|d\left(\eta^{1}\right)-d\left(\eta^{2}\right)\right|_{D} \leq C\left|\eta^{1}-\eta^{2}\right|_{R} .
$$

This completes the proof.

We point out that the approach taken in the proof of Lemma 6.4 is related to the technique utilized in [30, Section 3-4].

Once we have established the strong regularity of (6.1) at $d^{*}$, the proof of Theorem 6.3 , i.e., the locally quadratic convergence rate for (6.2), follows from [1, 12]; see also [30, Theorem 3.3]. Since the Newton process (6.2) is equivalent to the SQPiteration in Algorithm 4.1 we readily obtain the same locally quadratic convergence rate for $\left\{\left(x^{n}, p^{n}\right)\right\}$ produced by Algorithm 4.1.

REMARK 6.5. The smallness assumption imposed on $J_{y}\left(y^{*}, u^{*}\right)$ is commonly used in the literature; see [24] and the references therein. In the case of tracking-type functionals it can be guaranteed in the case of exact (or $\epsilon$ ) controllability of the desired state; compare [8, 13]. 
7. Numerical experiments. The control problem considered here is the tracking of the Stokes flow $z$ in a cavity; see Figure 7.1. Its formulation is given by $(\mathrm{P})$ with the cost functional

$$
J(y, u):=\frac{1}{2} \int_{Q}|y-z|^{2} d x d t+\frac{\alpha}{2} \int_{Q}|u|^{2} d x d t
$$

Here, $Q:=(0, T) \times \Omega$ with $\Omega:=(0,1)^{2}$ and $T:=1$. The desired state $z(t, x)=$ $\left(z_{1}, z_{2}\right)^{\top}$ is chosen such that $z(t, \cdot)=s(\cdot)$ for every time instance $t \in(0, T)$, where $s=\left(s_{1}, s_{2}\right)^{\top}$ denotes the stationary Stokes flow in the domain $\Omega$ with inhomogeneous boundary condition $s_{1}=1, s_{2}=0$ on $\partial \Omega$. The first term in the cost functional values the control gain when tracking the state $z$, and the second term measures the control cost, where $\alpha>0$ denotes a weighting factor. The value of the kinematic viscosity is $\nu=\frac{1}{10}$, and the initial velocity is $y_{0}=(0,0)^{\top}$.

The initial value of the uncontrolled flow is chosen as

$$
y(0, x)=e\left[\begin{array}{c}
-\left(\cos 2 \pi x_{1}-1\right) \sin 2 \pi x_{2} \\
\left(\cos 2 \pi x_{2}-1\right) \sin 2 \pi x_{1}
\end{array}\right]
$$

with $e$ denoting the Euler number. Note that $y(0, \cdot)_{\mid \partial \Omega}=(0,0)^{\top}$.

For the results presented an equidistant time discretization is chosen with step length $\delta t=0.01$, and for the spatial discretization the Taylor-Hood finite element [25] is used on a grid containing 256 triangles with 545 velocity and 145 pressure nodes. Including the primal, adjoint and control variables, the number of unknowns in the discretized control problem is $1.64 \times 10^{5}$. For the control problems considered in the subsequent examples finer grids yield similar results.

We note that in our approach the control variables are not discretized explicitly but implicitly through the first order optimality conditions. See [23] for an account for this discretization technique. We further point out that each part of the overall solution algorithm is discretized separately. Utilizing this technique, we obtain approximations of the reduced gradient and of 'reduced Hessian times increment' operations with respective approximation errors of the order of the discretization error of the Navier-Stokes equations (see [9] for an analysis of the latter type of error). As a consequence, it is only meaningful to monitor fast convergence of the SQP-framework of Algorithm 4.1 for stopping tolerances of the order of the discretization error of the state equations. Finally let us note that the active set is resolved on the grid induced by the velocity nodes (vertices of the triangulation together with edge midpoints).

In the sequel all iterates represent discrete quantities. The termination criterion for the outer SQP-iteration in Algorithm 4.1 is chosen as $\left|\left(\delta x^{n}, \delta p^{n}\right)\right| \leq$ tol $_{\mathrm{SQP}}:=$ $5 \times 10^{-3}$. Here $|\cdot|$ is the norm on $W \times U \times Z$. Note that the results in [9] yield a discretization error of $\mathcal{O}(0.01)$ for our discretization described above. The effect of our choice for tol $\mathrm{SQP}_{\mathrm{SP}}$ in connection with the discretization error is discussed in detail below. In iteration $n$, the stopping tolerance in Algorithm 5.1 is chosen as $\epsilon=$ tol $^{n}$ with

$$
\operatorname{tol}^{n}=\min \left\{\left|\left(\delta x^{n}, \delta p^{n}\right)\right|^{2}, 0.1\left|\Psi\left(\delta_{u, 0}, \hat{\lambda}_{0} ; u^{n}\right)-\hat{\lambda}_{0}\right|_{\left(L^{2}\right)^{2}}\right\} .
$$

Alternatively, we stop Algorithm 5.1 as soon as the active nodes of two successive iterations coincide. The inner CG-loop of Algorithm 5.1, i.e., Algorithm 5.2, is terminated if the iterate $v_{k}$ satisfies

$$
\left|\left(H^{n} v_{k}-r^{n}\right)_{\mathcal{I}_{l}}\right|_{\left(L^{2}\right)^{2}}<\min \left\{0.01\left|\left(H^{n} v_{0}-r^{n}\right)_{\mathcal{I}_{l}}\right|_{\left(L^{2}\right)^{2}},\left|r_{\mathcal{I}_{l}}^{n}\right|_{\left(L^{2}\right)^{2}}^{3 / 2}\right\} .
$$


All computations were performed on a DELL ${ }^{T M}$ laptop computer with $1 \mathrm{Ghz}$ CPU. In the tables below, the column of CG-steps has to be read as follows: $l: b$ indicates that in the $l$ th pdas-iteration (Algorithm 5.1) $b$ cycles of Algorithm 5.2 are performed until its stopping criterion is met.

In all test runs state, control and adjoint of the equality constraint are initialized with zero. The Lagrange multiplier $\hat{\lambda}$ in the pdas-Algorithm 5.1 is initialized with the right hand side of system (OR). Subsequently we use

$$
q^{n}=\frac{\left|\left(\delta_{x}^{n}, \delta_{p}^{n}\right)\right|}{\left|\left(\delta_{x}^{n-1}, \delta_{p}^{n-1}\right)\right|^{2}}
$$

to study the convergence speed of the SQP-method and

$$
c^{n}=\left|\hat{\Psi}\left(\delta_{u}^{n}\right)+\alpha\left(u^{n}+\delta_{u}^{n}\right)-S^{n} \delta_{u}^{n}-t^{n}\right|_{\left(L^{2}\right)^{2}}
$$

to measure the residual in the complementarity system at iteration $n$.

Example 1: The desired state $z$ has nonhomogeneous boundary conditions and therefore can not be reached, since we prescribe homogenous boundary conditions in (1.1). In Figure 7.1 the desired flow together with the initial flow is shown.
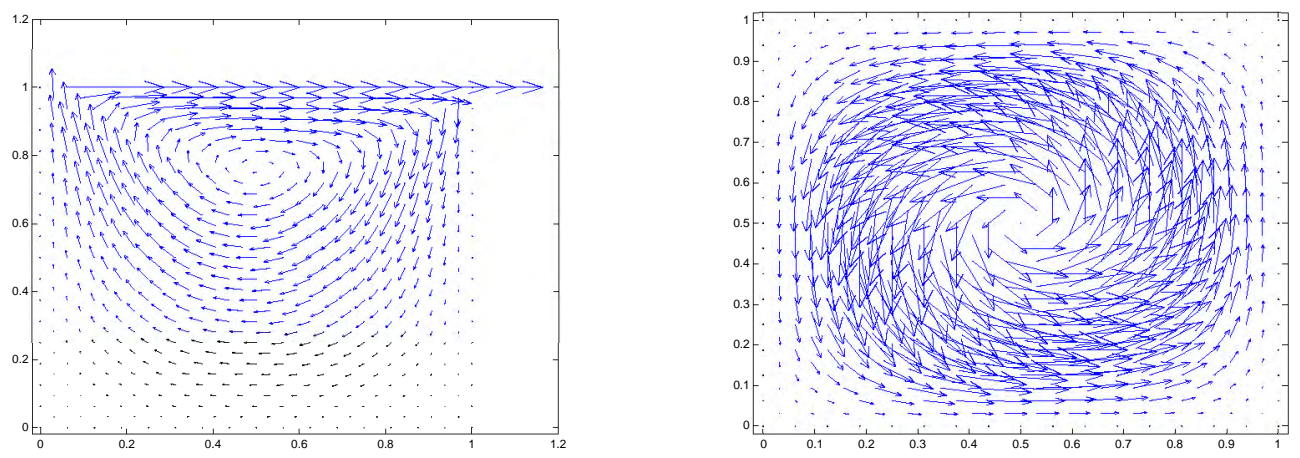

FIG. 7.1. Stationary Stokes flow $s$ in the cavity (left), and initial condition (right).

Table 7.1 presents the results for $\alpha=0.01$ and $a_{i}=-3, b_{i}=3$, in Table 7.2 the results for $\alpha=0.01$ and $a_{i}=-0.3, b_{i}=0.3$ are shown. The optimal value of the cost functional without constraints is $J^{*}=3.658206 \mathrm{e}-1$. ¿From the Tables 7.1-7.2 one can see that the active set is identified after at most 3 iterations of the primal-dual active set strategy (Algorithm 5.1). We recall that whenever the number of pdas-iterations is 2 then the active set is detected immediately, and pdAS stops successfully after the first cycle. This behavior is typical in our test runs also for other choices of the parameters involved in the optimization problem. Moreover we can study the impact of the discretization error on the convergence speed of the algorithm. Iterations 1-4 indicate the fast local convergence behavior. At iteration 4 the order of the discretization error is reached and subsequently the convergence speed is reduced; compare the last row in the $q^{n}$-column, respectively. We further study this effect in Example 2 below, where we compare the results for different mesh sizes.

Figure 7.2 shows the evolution of the cost functional as a function of time for different control boxes and $\alpha=0.01$. It displays the results for the uncontrolled, controlled but unconstrained, and constrained test runs. The controlled flow upon termination of our algorithm for $t=1$ and $t=0.06$ is shown in Figure 7.3. 

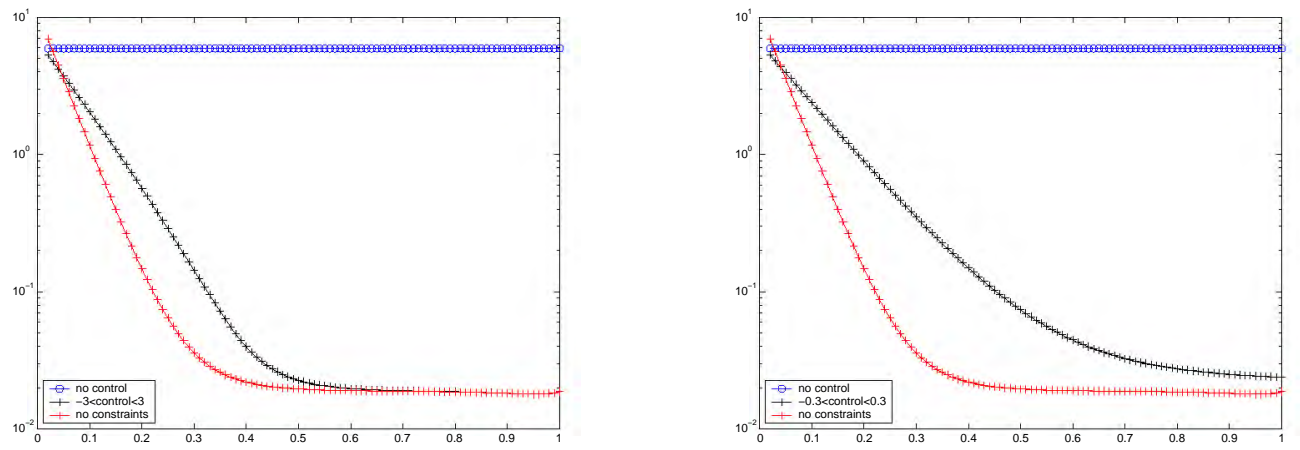

FIG. 7.2. Evolution of cost functional, $-3 \leq u_{i} \leq 3$ (left), $-0.3 \leq u_{i} \leq 0.3, \alpha=1$.E-2 (right).
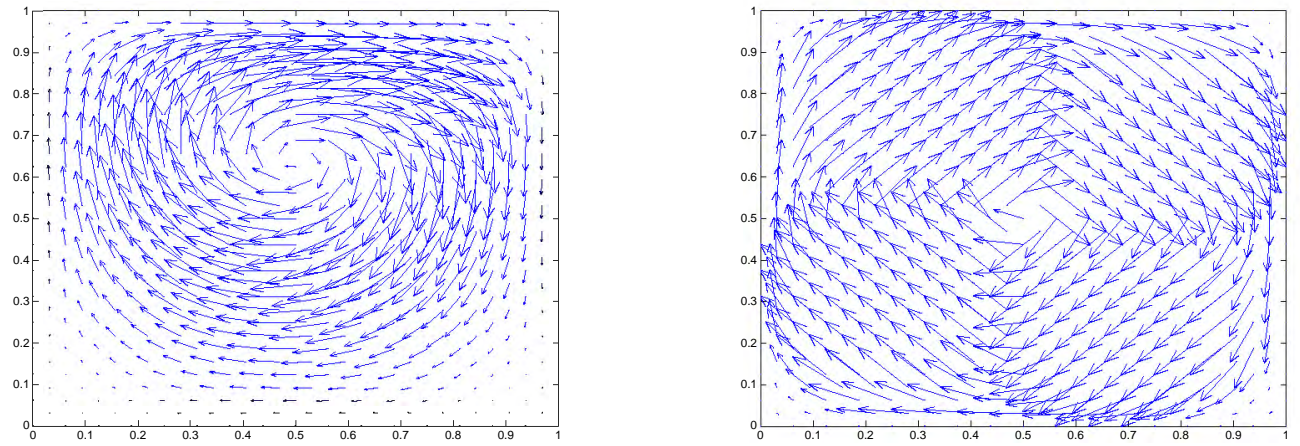

FIG. 7.3. Controlled flow at $t=1$ (left) and control at $t=0.06$ for (right) $-3 \leq u_{i} \leq 3$ and $\alpha=1 . \mathrm{e}-2$, Example 1 .

Example 2: The desired state $z$ is the same as in Example 1, but now we impose the boundary condition $y_{1}=1$ on $\Sigma$ in $(1.1)$. We note that $z(0, \cdot)_{\left.\right|_{\Omega}}=(1,0)^{\top}$ and $y_{0}=(0,0)^{\top}$ on $\partial \Omega$. Thus, as in Example 1 it is impossible to reach the desired state. However, we expect that the optimal value of the cost functional is significantly smaller than the one achieved in Example 1.

In Figure 7.4 the optimally controlled flow together with the corresponding optimal control at $t=0.75$ are shown. The evolution of the cost functional value is depicted in Figure 7.5.

As expected the value of the cost functional at the numerical solution is smaller than in Example 1, while the performance of the algorithm for both examples is very similar. This conclusions become evident upon studying Tables 7.3-7.4 and comparing the results with the ones given in Tables 7.1-7.2. The value of the cost functional without constraints for this example is $J^{*}=3.364230 \mathrm{e}-1$. To further illustrate the effect of the discretization error on the convergence speed, in Table 7.5 we also present our numerical findings for a spatial discretization using 2113 velocity and 545 pressure nodes on a time grid with $\delta t=0.00625$. The discretization error now is of the order $\mathcal{O}(6.25 \mathrm{e}-3)$ (compared to $\mathcal{O}(0.01)$ in the previous run). As for the run documented in Table 7.4 we choose $\alpha=0.01$ and $a_{i}=-0.3, b_{i}=0.3$. Note that the numerically observed convergence speed increases slightly compared to the one on the coarser grid presented in Table 7.4.

Finally, Figure 7.6 presents the active sets of Example 2 for $\alpha=0.01$ and $a_{i}=$ 


\begin{tabular}{|l|l|l|l|l|l|}
\hline Iteration & pdas-steps & CG-steps & $q^{n}$ & $c^{n}$ & $J\left(x^{n}\right)$ \\
\hline \hline 1 & 3 & $1: 4,2: 5,3: 0$ & $2.59 \mathrm{e}-1$ & 0 & $5.850577 \mathrm{e} 0$ \\
2 & 3 & $1: 10,2: 6,3: 0$ & $1.22 \mathrm{e}-1$ & $4.267064 \mathrm{e}-5$ & $4.848998 \mathrm{e}-1$ \\
3 & 2 & $1: 4,2: 0$ & $4.05 \mathrm{e}-1$ & $1.859095 \mathrm{e}-5$ & $4.796364 \mathrm{e}-1$ \\
4 & 2 & $1: 5,2: 0$ & $1.12 \mathrm{e}-2$ & $5.662859 \mathrm{e}-6$ & $4.700816 \mathrm{e}-1$ \\
\hline 5 & 2 & $1: 5,2: 0$ & $6.68 \mathrm{e} 0$ & $9.359857 \mathrm{e}-8$ & $4.700874 \mathrm{e}-1$ \\
\hline
\end{tabular}

Performance of Algorithm 4.1 for $\alpha=1$. -2 2 and $a_{i}=-3, b_{i}=3$, Example 1

\begin{tabular}{|l|l|l|l|l|l|}
\hline Iteration & pdas-steps & CG-steps & $q^{n}$ & $c^{n}$ & $J\left(x^{n}\right)$ \\
\hline \hline 1 & 2 & $1: 3,2: 0$ & $3.35 \mathrm{e}-1$ & $1.388674 \mathrm{e}-4$ & $5.850577 \mathrm{e} 0$ \\
2 & 2 & $1: 3,2: 0$ & $1.08 \mathrm{e}-1$ & $9.596955 \mathrm{e}-5$ & $5.969585 \mathrm{e}-1$ \\
3 & 2 & $1: 3,2: 0$ & $2.54 \mathrm{e}-1$ & $3.339041 \mathrm{e}-5$ & $5.826340 \mathrm{e}-1$ \\
4 & 2 & $1: 3,2: 0$ & $1.12 \mathrm{e}-1$ & $4.869688 \mathrm{e}-7$ & $5.820019 \mathrm{e}-1$ \\
\hline 5 & 2 & $1: 3,2: 0$ & $47.26 \mathrm{e} 0$ & $3.597841 \mathrm{e}-9$ & $5.820095 \mathrm{e}-1$ \\
\hline \multicolumn{5}{|c|}{ TABLE 7.2} \\
\hline
\end{tabular}

Performance of Algorithm 4.1 for $\alpha=1$. -2 and $a_{i}=-0.3, b_{i}=0.3$, Example 1

\begin{tabular}{|l|l|l|l|l|l|}
\hline Iteration & pdas-steps & CG-steps & $q^{n}$ & $c^{n}$ & $J\left(x^{n}\right)$ \\
\hline \hline 1 & 3 & $1: 4,2: 5,3: 0$ & $2.54 \mathrm{e}-1$ & 0 & $5.850577 \mathrm{e} 0$ \\
2 & 3 & $1: 11,2: 6,3: 0$ & $1.60 \mathrm{e}-1$ & $5.071075 \mathrm{e}-4$ & $4.537418 \mathrm{e}-1$ \\
3 & 2 & $1: 5,2: 0$ & $3.21 \mathrm{e}-1$ & $2.344538 \mathrm{e}-4$ & $4.549137 \mathrm{e}-1$ \\
4 & 2 & $1: 6,2: 0$ & $2.05 \mathrm{e}-2$ & $1.965041 \mathrm{e}-5$ & $4.317132 \mathrm{e}-1$ \\
\hline 5 & 2 & $1: 7,2: 0$ & $5.94 \mathrm{e}-1$ & $2.164409 \mathrm{e}-7$ & $4.316904 \mathrm{e}-1$ \\
\hline
\end{tabular}

Performance of Algorithm 4.1 for $\alpha=1$.e-2 and $a_{i}=-3, b_{i}=3$, Example 2

\begin{tabular}{|l|l|l|l|l|l|}
\hline Iteration & pdas-steps & CG-steps & $q^{n}$ & $c^{n}$ & $J\left(x^{n}\right)$ \\
\hline \hline 1 & 2 & $1: 3,2: 0$ & $3.25 \mathrm{e}-1$ & $1.388673 \mathrm{e}-4$ & $5.850577 \mathrm{e} 0$ \\
2 & 3 & $1: 3,2: 3,3: 0$ & $1.08 \mathrm{e}-1$ & $1.608579 \mathrm{e}-5$ & $5.410821 \mathrm{e}-1$ \\
3 & 3 & $1: 4,2: 4,3: 0$ & $3.00 \mathrm{e}-1$ & $1.044886 \mathrm{e}-5$ & $5.274123 \mathrm{e}-1$ \\
4 & 2 & $1: 4,2: 0$ & $2.70 \mathrm{e}-1$ & $7.775520 \mathrm{e}-6$ & $5.265481 \mathrm{e}-1$ \\
\hline 5 & 2 & $1: 4,2: 0$ & $2.72 \mathrm{e} 0$ & $4.319744 \mathrm{e}-8$ & $5.265512 \mathrm{e}-1$ \\
\hline
\end{tabular}

Performance of Algorithm 4.1 for $\alpha=1$.e-2 and $a_{i}=-0.3, b_{i}=0.3$, Example 2.

\begin{tabular}{|l|l|l|l|l|l|}
\hline Iteration & pdas-steps & CG-steps & $q^{n}$ & $c^{n}$ & $J\left(x^{n}\right)$ \\
\hline \hline 1 & 3 & $1: 3,2: 3,3: 0$ & $3.22 \mathrm{e}-1$ & $6.477752 \mathrm{e}-8$ & $5.877315 \mathrm{e} 0$ \\
2 & 3 & $1: 3,2: 3,3: 0$ & $1.08 \mathrm{e}-1$ & $1.824281 \mathrm{e}-5$ & $5.463363 \mathrm{e}-1$ \\
3 & 3 & $1: 4,2: 4,3: 0$ & $3.07 \mathrm{e}-1$ & $1.446313 \mathrm{e}-5$ & $5.336359 \mathrm{e}-1$ \\
4 & 2 & $1: 4,2: 0$ & $2.78 \mathrm{e}-1$ & $1.077478 \mathrm{e}-5$ & $5.327380 \mathrm{e}-1$ \\
\hline 5 & 2 & $1: 6,2: 0$ & $1.19 \mathrm{e} 0$ & $4.802076 \mathrm{e}-8$ & $5.327348 \mathrm{e}-1$ \\
\hline
\end{tabular}

Performance of Algorithm 4.1 for $\alpha=1$. -2 and $a_{i}=-0.3, b_{i}=0.3$, Example 2, finer time and spatial grid. 

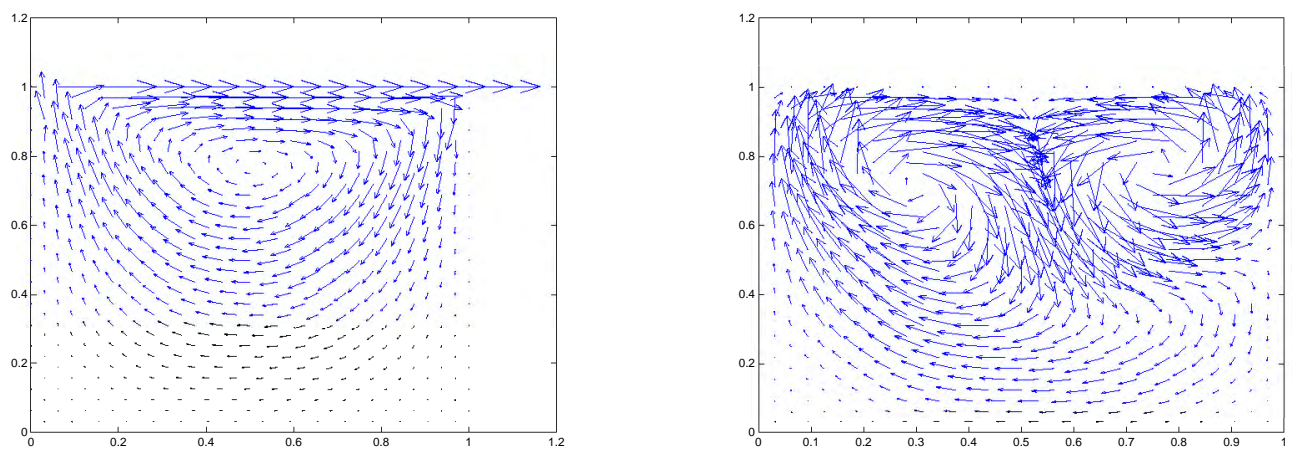

FIG. 7.4. Optimally controlled flow (left) together with control at $t=0.75$ for $-3 \leq u_{i} \leq 3$, Example 2.
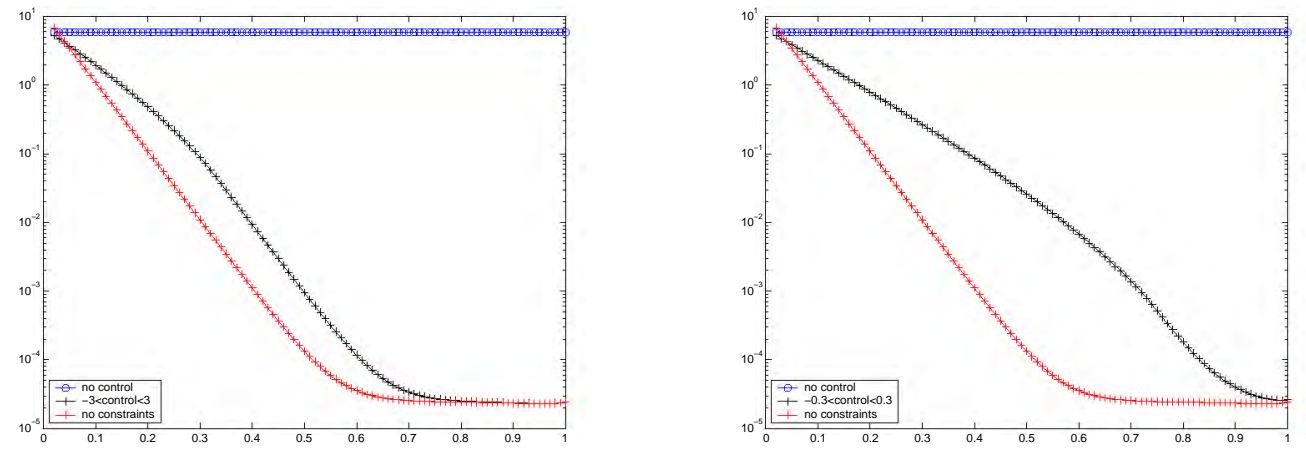
1.e-2

FIG. 7.5. Evolution of cost functional for $-3 \leq u_{i} \leq 3$ (left), and $-0.3 \leq u_{i} \leq 0.3$ (right), $\alpha=$

$-0.3, b_{i}=0.3$ at times $t=0.6$ (left) and $t=0.72$ (right). Here the entry ' + ' indicates that at least one of the control components is equal to one of the bounds in $U_{\mathrm{ad}}$. The figures indicate that with increasing time the number of active nodes decreases, which is exactly the behavior one would expect. In fact, due to our choice of initial conditions and desired state there is a strong control action at the beginning of the process. This action eventually decays since the controlled state gets closer to the desired profile as time evolves. Note that the left picture presents the active nodes of the control shown in the right picture of Figure 7.7.

Acknowledgements. The second author acknowledges the support of the Sonderforschungsbereich 609 Elektromagnetische Strömungsbeeinflussung in Metallurgie, Kristallzüchtung und Elektrochemie funded by the Deutsche Forschungsgemeinschaft.

\section{REFERENCES}

[1] W. Alt. The Lagrange-Newton method for infinite-dimensional optimization problems. Numer. Funct. Anal. and Optim., 11:201-224, 1990.

[2] H. Amann. Compact embeddings of vector-valued Sobolev and Besov spaces. Glas. Mat., III. Ser., 35(1):161-177, 2000.

[3] M. Berggren. Numerical solution of a flow-control problem: vorticity reduction by dynamic boundary action. SIAM J. Sci. Comput., 19:829-860, 1998. 

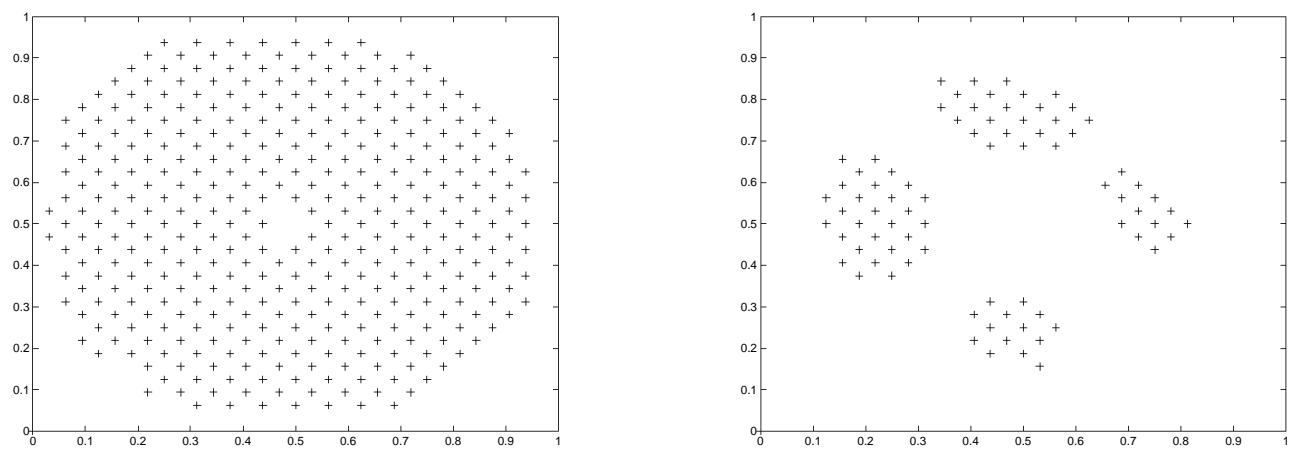

Fig. 7.6. Active set at $t=0.60$ (left) and at $t=0.72$ (right), Example 2.
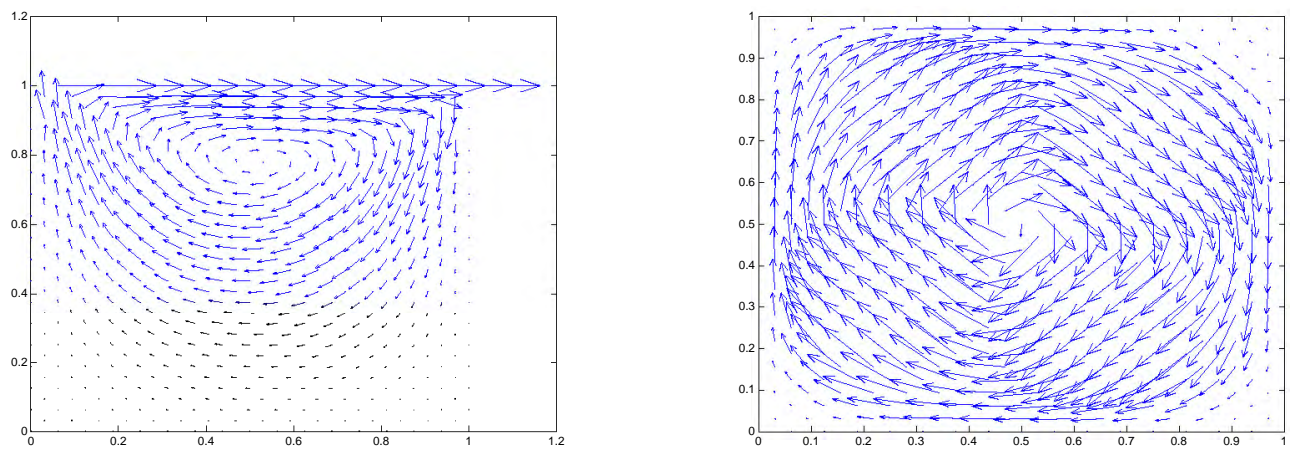

FIG. 7.7. Optimally controlled flow (left) together with control at $t=0.6$ for $-0.3 \leq u_{i} \leq 0.3$, Example 2.

[4] M. Bergounioux, K. Ito, and K. Kunisch. Primal-dual strategy for constrained optimal control problems. SIAM J. Control and Optim., 37:1176-1194, 1999.

[5] M. Bergounioux, M. Haddou, M. Hintermüller, and K. Kunisch. A comparison of a MoreauYosida based active set strategy and interior point methods for constrained optimal control. SIAM J. Optimization, 11:495-521, 2000.

[6] T.R. Bewley. Flow control: new challenges for a new rennaissance. Progress in Aerospace Sciences, 37:21-58, 2001.

[7] X. Chen, Z. Nashed, and L. Qi. Smoothing methods and semismooth methods for nondifferentiable operator equations. SIAM J. Num. Anal., 38:1208-1216, 2000.

[8] F. Colonius, and K. Kunisch. Output least squares stability for parameter estimation in two point value problems. Journal für die Reine und Angewandte Mathematik, 370:1-29, 1986.

[9] K. Deckelnick, and M. Hinze. Error estimates in space and time for tracking-type control of the instationary Stokes system. ISNM, 143:87-103, 2002.

[10] R. Dembo, S. Eisenstat, and T. Steihaug. Inexact Newton methods. SIAM J. Numer. Anal., 19:400-408, 1982.

[11] M. Desai, and K. Ito. Optimal controls of Navier-Stokes equations. SIAM J. Control Optim., 32:1428-1446, 1994.

[12] A. Dontchev. Local analysis of a Newton-type method based on partial linearization. In Proceedings of the AMS-SIAM Summer Seminar in Applied Mathematics on Mathematics and Numerical Analysis: Real Number Algorithms, J. Renegar, M. Shub, and S. Smale, eds, AMS Lectures in Appl. Math., 32:295-306, 1996.

[13] A. L. Dontchev, and T. Zolezzi. Well-posed Optimization Problems. Springer, Lecture Notes in Mathematics, 1543, Berlin 1993.

[14] O. Ghattas, and J. Bark. Optimal control of two- and three-dimensional incompressible NavierStokes flow. J. Comp. Phys., 136:231-244, 1997.

[15] S. Eisenstat, and H. Walker. Choosing the forcing terms in an inexact Newton method. SIAM 
J. Sci. Comput., 17:16-32, 1996.

[16] R. Glowinski. Finite element methods for the numerical simulation of incompressible viscous flow. Introduction to the Control of the Navier-Stokes Equations. Lect. Appl. Math., 28 (1991).

[17] M. Gunzburger, and S. Manservisi. Analysis and approximation of the velocity tracking problem for Navier-Stokes flows with distributed control. SIAM J. Numer. Anal., 37:1481-1512, 2000.

[18] M. Heinkenschloss. Formulation and analysis of a sequential quadratic programming method for the optimal Dirichlet boundary control of Navier-Stokes flow. In Optimal Control: Theory, Algorithms and Applications, Kluwer Academic Publishers B.V., 1998, pp. 178-203.

[19] M. Hintermüller. A primal-dual active set algorithm for bilaterally control constrained optimal control problems. Quarterly of Applied Mathematics, LXI: 131-161, 2003.

[20] M. Hintermüller. On a globalized augmented Lagrangian-SQP algorithm for nonlinear optimal control problems with box constraints. In Fast solution methods for discretized optimization methods, K.H. Hoffmann, R. Hoppe, and V. Schulz Eds., Internat. Ser. Numer. Math., 138 (2001), pp. 139-153.

[21] M. Hintermüller, and M. Hinze. Globalization of SQP-methods in control of the instationary Navier-Stokes equations. Mathematical Modelling and Numerical Analysis (M2AN), 36:725-746, 2002.

[22] M. Hintermüller, K. Ito, and K. Kunisch. The primal-dual active set strategy as a semismooth Newton method. SIAM J. Optimization, 13:865-888, 2003.

[23] M. Hinze. A generalized discretization concept for optimal control problems with control constraints. Preprint MATH-NM-02-2003, Institut für Numerische Mathematik, Technische Universität Dresden, 2003.

[24] M. Hinze, and K. Kunisch. Second order methods for optimal control of time-dependent fluid flow. SIAM J. Optim and Control, 40:925-946, 2001.

[25] P. Hood and C. Taylor. A numerical solution of the Navier-Stokes equations using the finite element technique. Comp. and Fluids, 1:73-100, 1973.

[26] J.L. Lions. Control of Distributed Singular Systems. Gauthier-Villars publisher, Paris, 1985.

[27] S.M. Robinson. Strongly regular generalized equations. Math. of Operation Research. 5:43-62, 1980.

[28] R. Temam. Navier-Stokes Equations. Series Studies in Mathematics and its Applications, vol. 2, 2nd edition, North-Holland publishers, Amsterdam, 1985.

[29] H. Triebel. Interpolation Theory, Function Spaces, Differential Operators. J.A. Barth-Verlag, 2nd edition, Heidelberg/Leipzig, 1995.

[30] F. Tröltzsch, S. Volkwein. The SQP method for control constrained optimal control of the Burgers equation. ESAIM: Control, Optimization and Calculus of Variations, 6:649-674, 2001.

[31] M. Ulbrich. Constrained optimal control of Navier-Stokes flow by semismooth Newton methods. Systems and Control Letters, 48:297-311, 2003.

[32] M. Ulbrich. Semismooth Newton methods in function spaces. SIAM J. Optimization, 13:805$842,2003$. 